\title{
THE
}

\section{Partial Averaging Approach to Fourier Coefficient Path Integration}

Rob D. Coalson

David L. Freeman

University of Rhode Island, dfreeman@uri.edu

Jimmie D. Doll

Follow this and additional works at: https://digitalcommons.uri.edu/chm_facpubs

Terms of Use

All rights reserved under copyright.

\section{Citation/Publisher Attribution}

Coalson, R. D., Freeman, D. L., \& Doll, J. D.. (1986). Partial Averaging Approach to Fourier Coefficient Path Integration. J. Chem. Phys., 85(8), 4567-4583. doi: 10.1063/1.451778

Available at: http://dx.doi.org/10.1063/1.451778

This Article is brought to you for free and open access by the Chemistry at DigitalCommons@URI. It has been accepted for inclusion in Chemistry Faculty Publications by an authorized administrator of DigitalCommons@URI. For more information, please contact digitalcommons@etal.uri.edu. 


\title{
Partial averaging approach to Fourier coefficient path integration
}

Rob D. Coalsona)

Theoretical Division, Los Alamos National Laboratory, Los Alamos, New Mexico 87545

David L. Freeman

University of Rhode Island, Department of Chemistry, Kingston, Rhode Island 02881

Jimmie D. Doll

Chemistry Division, Los Alamos National Laboratory, Los Alamos, New Mexico 87545

(Received 7 May 1986; accepted 19 June 1986)

\begin{abstract}
The recently introduced method of partial averaging is developed into a general formalism for computing simple Cartesian path integrals. Examples of its application to both harmonic and anharmonic systems are given. For harmonic systems, where analytical results can be derived, both imaginary and complex time evolution is discussed. For two representative anharmonic systems, Monte Carlo path integral simulations of the imaginary time propagator (statistical density matrix) are presented. Connections with other Cartesian path integral techniques are stressed.
\end{abstract}

The major source of difficulty in calculating properties of many chemically interesting systems is easy to pinpoint. Chemical systems, governed as a rule by the nonrelativistic Schrödinger equation, typically involve many degrees of freedom whose motion is coupled in a complicated fashion. Unfortunately, when approached via traditional solution techniques, the Schrödinger equation becomes rapidly intractable with increasing spatial dimensionality. Again, the reason is simple: The traditional approach entails solution of the time independent Schrödinger equation $H \Psi=E \Psi$ via a basis set expansion. A conservative estimate of the number of basis functions needed to converge results for a coupled $d$ degree of freedom system is $N^{d}$, where $N$ is the number of basis functions necessary to converge results for a generic one-dimensional problem. In other words, the basis set size grows geometrically with dimension. Thus, the efficacy of basis function techniques is limited to problems with only a few degrees of freedom.

In the past few years it has become increasingly apparent that Feynman's path integral formulation of quantum mechanics, ${ }^{1}$ when combined with Monte Carlo sampling techniques, ${ }^{2}$ offers a way out of the basis set dilemma described above in at least some interesting cases. More precisely, the path integral formalism focuses on the evaluation of the coordinate space propagator, $\left\langle\mathbf{x}_{f}|\exp (-\kappa \hat{H})| \mathbf{x}_{0}\right\rangle$ $\equiv \rho_{\kappa}\left(\mathbf{x}_{f}, \mathbf{x}_{0}\right)$, where the parameter $\kappa$ depends on the property of interest. For example, if $\kappa=1 / k_{B} T$ with $k_{B}$ Boltzmann's constant and $T$ the system temperature, then $\int d \mathbf{x} \rho_{\beta}(\mathbf{x}, \mathbf{x})=Z_{\beta}$, the quantum partition function for a system of distinguishable particles. From the partition function all thermodynamic properties can be calculated. If $\kappa=i t / \hbar$, then $\rho_{i t / \hbar}$ can be used to evolve an arbitrarily prepared initial wave packet to time $t$. From such temporal evolution scattering cross sections, spectra and rate constants of various kinds can be extracted. Indeed, in a formal sense $\rho_{\kappa}$ may be regarded as a holy grail: Knowledge of $\rho_{\kappa}$ enables computation of essentially any quantum mechanical observable.

The critical issue, clearly, is one of implementability.

\footnotetext{
a) Present address: Department of Chemistry, University of Pittsburgh, Pittsburgh, PA 15260.
}

Fortunately, the path integral formalism enables numerical computation of $\rho_{\kappa}$ in favorable circumstances. Moreover, these circumstances as a rule have relatively little to do with the spatial dimensionality of the problem under consideration. In particular, the path integral (PI) formulation reduces the calculation of $\rho_{\kappa}$ to an exercise in multidimensional integration. If the relevant integrals were attempted by conventional quadrature schemes, the "basis set dilemma" would recur: The number of quadrature points needed in such schemes goes up geometrically with the dimensionality of the integration space. However, by using importance sampling techaniques to perform the integrals it is frequently the case that substantial dimensionality increases require only mild increases in computational effort. Thus, if a particular property can be computed by Monte Carlo path integration (MCPI) for a 1d system, it can be computed for a many degree of freedom system without increasing the numerical effort by orders of magnitude.

As impressive as importance sampling techniques are, it is clearly unwise to tempt the hand of fate with regard to their convergence properties: Any reduction in the dimensionality of the requisite integrals and/or the complexity of the integrands involved is to be welcomed. A number of attempts along these lines have now been made. Most have concentrated on improving the small argument propagator expression from which finite argument propagators are synthesized.

Suzuki ${ }^{3}$ has given a formal procedure for constructing improved small argument propagators. De Raedt and De Raedt ${ }^{4}$ have extended and applied Suzuki's ideas, mainly to problems of interest in solid state physics. In the arena of chemical physics, Thirumalai and Berne ${ }^{5}$ and Schweizer et $a l .^{6}$ have discussed schemes for improving the accuracy of the small argument propagator for systems governed by Cartesian coordinate space Hamiltonians.

Recently, we approached the convergence issue for simple Cartesian coordinate space path integrals in a different way. ${ }^{7}$ Specifically, the formally exact Fourier coefficient prescription for such PI's was used as a point of departure. From here, certain connections between PI quantum mechanics and Brownian motion theory were exploited in order 
to average out the rapid fluctuations in typical ("Brownian") paths encountered when "summing over paths" in accordance with the PI formalism. This resulted in an implementable procedure, designated as "partial averaging," which was essentially the original Fourier coefficient prescription with the system potential replaced by an appropriate effective potential. Partial averaging (PA) was found to enhance convergence of MCPI simulations dramatically for a 1d double well potential problem. A number of other examples have now been examined; all have displayed the same favorable trends upon application of PA. Moreover, a number of promising ramifications of PA theory have been developed. For these reasons, a fuller exposition of the PA prescription seems warranted. An outline of this exposition follows.

We will divide our discussion into three parts: Theory, applications, and speculations. Theoretical development will be presented in a sequence of six brief sections. The essential features of partial averaging are introduced in Sec. I, where evaluation of the imaginary time propagator $\rho_{\beta}$ in $1 \mathrm{~d}$ is considered. In Sec. II the PA procedure is explicitly extended to the case of multidimensional coupled potentials. It is important to make this extension clear, since the ultimate goal of MCPI techniques is to be able to treat such complicated systems. In Sec. III the underlying cumulant structure of PA is stressed. This serves to elevate PA from an approximation procedure to an exact formalism which naturally suggests the basic PA prescription presented in Secs. I and II as a first level of approximation. With the groundwork laid in Sec. III, it is then possible in Sec. IV to extend PA to the important case of mixed time propagation $[\beta \rightarrow \beta+i t / \hbar]$. Sections $V$ and VI further utilize the cumulant structure of PA to derive "improved small argument propagator" results, and make connections with similar results obtained by other workers. In Sec. V the effective small argument potential arrived at by Schweizer $e t$ al. using a Migdal transformation is derived in a very simple way via PA. In Sec. VI this effective potential is shown to be exact through $O\left(\epsilon^{2}\right), \epsilon$ being the relevant "small argument" parameter. All terms contributing through $O\left(\epsilon^{2}\right)$ are extracted.

In the second part of the paper a number of applications of the PA procedure are presented. These divide naturally into two classes: Those concerning harmonic systems and those concerning anharmonic systems. As is well known, harmonic oscillator systems provide an obvious first testing ground for new PI methods. All integrals arising in connection with path integration of harmonic oscillator systems can be performed analytically. This removes problems concerning numerical precision of the quadrature schemes and importance sampling techniques which must be employed in order to treat more general systems. It is then possible to see, if one is working with a Fourier coefficient framework, how many coefficients are needed to describe the paths which enter into the Feynman "sum over paths." The fewer the number of Fourier coefficients needed, the more efficient a particular PI algorithm is.

We have found that PA dramatically improves the efficiency with which harmonic oscillator systems can be treated. A number of cases are discussed in Sec. VII. Partition functions for various temperature systems are computed before and after partial averaging. The results are compared with analogs obtained using discretized PI methods. The second cumulant correction to the PA prescription is also computed. It is seen to further enhance the quality of the PA results. Finally, to demonstrate the promise of the PA prescription for treating complex time systems, two problems of current interest are considered. Namely, thermally averaged transmission coefficients ("reaction rates") for tunneling through an inverted parabolic barrier are computed using the propagator based formalism of Miller, Schwartz, and Tromp. ${ }^{8}$ In addition, electronic absorption spectra for two linearly displaced harmonic wells are calculated via the traditional time kernel formalism as adapted recently to enable utilization of propagator techniques. ${ }^{9}$ In both these cases, partial averaging uniformly and significantly enhances the accuracy of the results.

Section VIII presents a number of imaginary time results concerning two anharmonic potentials, a Morse well and a double well. For these systems MCPI simulations were performed to numerically enumerate the appropriate sum over paths. For both systems, trends similar to those observed in the harmonic oscillator problems discussed in Sec. II $A$ are seen. In particular, partial averaging uniformly and dramatically enhances the convergence rates of imaginary time propagator calculations.

The final part of the paper consists of a single section entitled Practical Considerations and the Role of Fractals in Path Integrations. The theme of this section is somewhat curious, since it attempts to embrace the mundane and the sublime simultaneously. Its purpose is correspondingly twofold: We wish to point out what appears to be the essential numerical advantage of the PA procedure. The desired results can be obtained by considering a small set of smooth paths which comprise a tiny subset of the paths formally included in the Feynman path integral prescription. Not only does PA enable converged results to be obtained with fewer integration variables than would otherwise be necessary, it also leads to smooth "integrals along the potential," which can then be evaluated with relatively few (expensive) calls to the potential function. On a more conceptual level, we wish to raise the possibility that the unusual properties of continuous but nondifferentiable Brownian paths play at most a minor role in determining evolution of systems governed by simple Cartesian Hamiltonians.

\section{1d PARTIAL AVERAGING IN IMAGINARY TIME}

Consider a 1d Hamiltonian of the form

$$
\hat{H}=\frac{\hat{p}^{2}}{2 m}+V(\hat{x}) \text {. }
$$

Note that position-momentum coupling is excluded by the form of Eq. (1.1). Only simple Cartesian Hamiltonians ("Cartesian kinetic energy plus position dependent potential") will be studied in this paper.

We start from the Fourier coefficient path integral (FPI) expression for the coordinate space propagator ${ }^{1,10,30}$ 


$$
\begin{aligned}
\frac{\rho_{\beta}\left(x_{f}, x_{0}\right)}{\rho_{\beta}^{f p}\left(x_{f}, x_{0}\right)}= & {\left[\prod_{k=1}^{\infty} 2 \pi \sigma_{k}^{2}\right]^{-1 / 2} \int d a_{1} \ldots . d a_{\infty} } \\
& \times \exp \left[-\sum_{k=1}^{\infty} a_{k}^{2} / 2 \sigma_{k}^{2}-\beta \int_{0}^{1} d u V(q(u))\right] .
\end{aligned}
$$

Here $\rho_{\beta}^{f}\left(x_{f}, x_{0}\right)$ is the free particle propagator

$\rho_{\beta}^{f_{P}}\left(x_{f}, x_{0}\right)=\left[\frac{m}{2 \pi \hbar^{2} \beta}\right]^{1 / 2} \exp \left[-\frac{m}{2 \hbar^{2} \beta}\left(x_{f}-x_{0}\right)^{2}\right]$,

$q(u)$ is the Fourier path

$$
q(u)=x_{0}+\left(x_{f}-x_{0}\right) u+\sum_{k=1}^{\infty} a_{k} \sin (k \pi u),
$$

and $\sigma_{k}^{2}$, the mean square deviation of $a_{k}$ from zero in the $V \rightarrow 0$ limit, is given by

$$
\sigma_{k}^{2}=\frac{2 \hbar^{2} \beta}{m(\pi k)^{2}} \text {. }
$$

Note that the Fourier coefficient formulation of the Cartesian Hamiltonian path integral has a natural and intuitively appealing length scale built into it. The more highly oscillatory sine wave contributions to a typical path are constrained to have smaller and smaller amplitudes according to Eq. (1.5). Thus, it seems reasonable to assume that by truncating Eq. (1.2) [and the associated Eq. (1.4)] after some modest number $k_{\max }$ of coefficients that a good approximation to the (formally exact) infinite coefficient result may be obtained. The hope is that by repeating the calculation for successively larger values of $k_{\max }$ the result will cease changing before $k_{\max }$ becomes so large that the calculation becomes unwieldy. This hope has now been borne out in a number of cases. ${ }^{10,11}$ However, it has at the same time become clear that for highly quantal (large $\beta$ ) systems the convergence rates can be frustratingly slow. The question arises as to whether a treatment can be devised which incorporates the major effect of the rapid fluctuations contributed by sine waves with $k>k_{\max }$ without explicitly considering individual higher order paths. This is the objective of the method of partial averaging.

Let us rewrite Eq. (1.2) in the following way:

$$
\begin{aligned}
\frac{\rho_{\beta}\left(x_{f}, x_{0}\right)}{\rho_{\beta}^{f p}\left(x_{f}, x_{0}\right)}= & {\left[\prod_{k=1}^{k_{\max }} 2 \pi \sigma_{k}^{2}\right]^{-1 / 2} } \\
& \times \int d a_{1} \ldots d a_{k_{\max }} \exp \left[-\sum_{k=1}^{k_{\max }} a_{k}^{2} / 2 \sigma_{k}^{2}\right] \\
& \times\left\langle\exp \left[-\beta \int_{0}^{1} d u V(q(u))\right]\right\rangle_{a_{k_{\max }+1, \ldots, a_{\infty}}},
\end{aligned}
$$

where \langle\rangle$_{a_{k_{\max }+1, \ldots, a_{\infty}}}$ means "average over the Gaussian variables $a_{k_{\max }+1}, \ldots, a_{\infty}$." Specifically,

$$
\begin{aligned}
\langle\exp & \left.-\beta \int_{0}^{1} d u V(q(u))\right\rangle_{a_{k_{\max }+1, \ldots, a_{\infty}}} \\
= & {\left[\prod_{k=k_{\max }+1}^{\infty} 2 \pi \sigma_{k}^{2}\right]^{-1 / 2} \int d a_{k_{\max }+1} \ldots d a_{\infty} } \\
& \times \exp -\left[\sum_{k=k_{\max }+1}^{\infty} a_{k}^{2} / 2 \sigma_{k}^{2}-\beta \int_{0}^{1} d u V(q(u))\right] .
\end{aligned}
$$

Equations (1.6) and (1.7) are, of course, completely equivalent to Eq. (1.2). The integration over all $a_{k}$ 's has simply been organized in a way which suggests the following approximation: Since the fluctuations in $a_{k_{\max }}+1, \ldots, a_{\infty}$ can be made arbitrarily small by choosing $k_{\max }$ large enough, we appeal to Gibbs' inequality ${ }^{15}$ :

$$
\begin{aligned}
& \left\langle\exp \left[-\beta \int_{0}^{1} d u V(q(u))\right]\right)_{a_{k_{\max }+1, \ldots, a_{\infty}}} \\
& \quad \geqslant \exp \left[-\beta \int_{0}^{1} d u\langle V(q(u))\rangle_{a_{k_{\max }+1}, \ldots, a_{\infty}}\right] .
\end{aligned}
$$

Upon replacing the left-hand side of Eq. (1.8) with the righthand side a considerable simplification arises, because the inifinite dimensional integral in the exponent of the righthand side of Eq. (1.8) can be reduced to a one-dimensional integral. Moreover, this integral can be performed analytically in many cases and to good approximation in virtually all cases.

It is well known from random variable theory that a linear combination of Gaussian random variables generates a single Gaussian random process, ${ }^{12}$ i.e.,

$$
\begin{aligned}
{\left[\prod_{k=1}^{N} 2 \pi \sigma_{k}^{2}\right]^{-1 / 2} \int d x_{1} \ldots d x_{N} } \\
\quad \times \exp \left[-\sum_{k=1}^{N} x_{k}^{2} / 2 \sigma_{k}^{2}\right] F\left(\sum_{k=1}^{N} \lambda_{k} x_{k}\right) \\
=\left[2 \pi \sigma^{2}\right]^{-1 / 2} \int d p e^{-p^{2} / 2 \sigma^{2}} F(p)
\end{aligned}
$$

with

$$
\sigma^{2}=\sum_{k=1}^{N} \lambda_{k}^{2} \sigma_{k}^{2}
$$

Applying this result to the average on the right-hand side of Eq. (1.8) we obtain

$$
\langle V(q(u))\rangle_{a_{k_{\max }+1, \ldots, a_{\infty}}}=V_{\text {eff }}\left(q_{k_{\max }}(u), u\right) .
$$

In Eq. $(1.10), q_{k_{\max }}(u)$ is a Fourier path composed of the $k_{\max }$ lowest sine waves in the appropriate Fourier series ex-

$$
q_{k_{\max }}(u)=x_{0}+\left(x_{f}-x_{0}\right) u+\sum_{k=1}^{k_{\max }} a_{k} \sin (k \pi u) .
$$

Furthermore, $V_{\text {eff }}$ is an effective potential obtained from $V$ through the Gaussian transform:

$$
\begin{aligned}
V_{\text {eff }} & \left(q_{k_{\max }}(u), u\right) \\
\quad= & {\left[2 \pi \sigma^{2}(u)\right]^{-1 / 2} \int d p e^{-p^{2} / 2 \sigma^{2}(u)} V\left(q_{k_{\max }}(u)+p\right), }
\end{aligned}
$$

where the width of the transform variable $p$ is given by

$$
\begin{aligned}
\sigma^{2}(u) & =\sum_{k=k_{\max }+1}^{\infty} \sigma_{k}^{2} \sin ^{2}(k \pi u) \\
& =\frac{\hbar^{2} \beta}{m} u(1-u)-\sum_{k=1}^{k_{\max }} \sigma_{k}^{2} \sin ^{2}(k \pi u) \\
& =\frac{\hbar^{2} \beta}{m}\left[u(1-u)-\frac{2}{\pi^{2}} \sum_{k=1}^{k_{\max }} \frac{\sin ^{2}(k \pi u)}{k^{2}}\right] .
\end{aligned}
$$


Note that $\sigma^{2}(u)$ depends on $k_{\max }$, although for notational simplicity this dependence has been suppressed. Equation (1.13b) shows that the effective fluctuations scale as the thermal de Broglie wavelength $\Lambda=\left[\beta \hbar^{2} / m\right]^{1 / 2}$. Some representative plots of the dimensionless function $\left[\sigma^{2}(u)\right]^{1 / 2} /$ $\Lambda$ which modulates this length scale are shown in Fig. 1.

For completeness, let us assemble the net partial averaging prescription:

$$
\begin{aligned}
\frac{\rho_{\beta}\left(x_{f}, x_{0}\right)}{\rho_{\beta}^{f}\left(x_{f}, x_{0}\right)} \geqslant & {\left[\prod_{k=1}^{k_{\max }} 2 \pi \sigma_{k}^{2}\right]^{-1 / 2} } \\
& \times \int d a_{1} \ldots d a_{k_{\max }} \exp \left[-\sum_{k=1}^{k_{\max }} a_{k}^{2} / 2 \sigma_{k}^{2}\right. \\
& \left.-\beta \int_{0}^{1} d u V_{\text {eff }}\left(q_{k_{\max }}(u), u\right)\right]
\end{aligned}
$$

Thus, the PA prescription is seen to be operationally identical to its canonical FPI analog except that the system potential has been replaced with an effective "time dependent" potential $V_{\text {eff }}$ for purposes of computing the contribution of a particular Fourier path to the path integral. It is apparent that the utility of Eq. (1.14) hinges upon our ability to compute $V_{\text {eff }}$ efficiently. Let us assert that $V_{\text {eff }}$ can be readily evaluated (this will be affirmed for a large collection of potentials in the following paragraphs). Under such circumstances the PA prescription requires hardly any more computational effort to implement than its canonical counterpart. However, the convergence enhancement is generally quite dramatic when PA is performed, as will be demonstrated in the applications sections. Moreover, it should be noted that if $V_{\text {eff }}$ is calculated exactly, PA supplies a strict lower bound to $\rho_{\beta}\left(x_{f}, x_{0}\right)$ for any $x_{0}, x_{f}$. Therefore numerous integrals over $\rho_{\beta}$ (e.g., the partition function) yield strict bounds as well.

Before discussing the details of evaluating $V_{\text {eff }}$, it is appropriate to dwell briefly on the physical motivation behind partial averaging. Consider the task of evaluating the basic FPI expression, Eq. (1.2). To do so typically implies that some means of truncating the infinite dimensional integral indicated there must be devised. Since $\sigma_{k}^{2} \sim k^{-2}$, it is plausi-

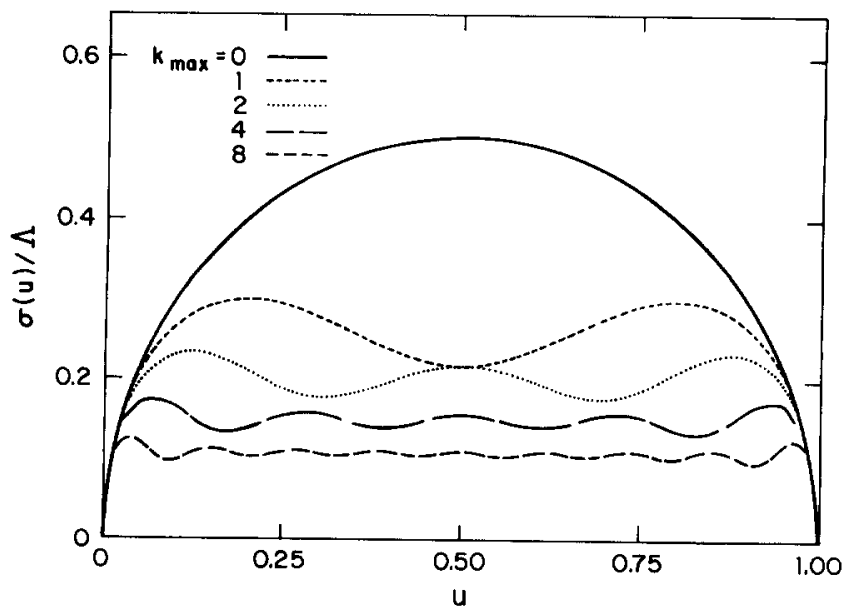

FIG. 1. Plots of reduced Gaussian transform width $\left[\sigma^{2}(u)\right]^{1 / 2} / \Lambda$ [cf. Eq. (1.13) vs $u$ ] for $k_{\max }=0,1,2,4,8$. ble to argue that for many interaction potentials of interest the high order fluctuations with Fourier index greater than some maximum value $k_{\max }$ become sufficiently small that they are effectively free-particle-like. If this is so, then $\left\langle a_{k}\right\rangle=0$ for $k>k_{\max }$, and we might argue that it is reasonable to replace $V(q(u))$ in Eq. (1.2) by $V\left(q_{k_{\max }}(u)\right)$. That is, we argue that the cumulative effect of including all high order fluctuations will vanish. This is the basic FPI method.

The partial averaging method also utilizes the "smallness" of the high order fluctuations, but in a less drastic manner. In the PA approach we retain both first and second moment information concerning the high order (free-particle-like) fluctuations. Thus, although for $k>k_{\max }\left\langle a_{k}\right\rangle=0$, $\left\langle a_{k}^{2}\right\rangle=\sigma_{k}^{2}$. This suggests that the effects of the high order terms can be "projected" onto the low order paths and can be included (approximately) by retaining only a small amount of additional information concerning their fluctuations. This line of development ultimately leads us to view each low order path as not simply a single path, but as representing a family of paths with a common low order "core" and all possible high order (free-particle) fluctuations. The contribution of each low order path is thus no longer simply the integral of the (bare) potential along that path, but becomes in the PA method the integral along that path of the potential averaged over the high order (free-particle) fluctuations [Eqs. (1.12)-(1.13)]. Thus, in the spirit of the effective potential formalism of Feynman and Hibbs, ${ }^{1}$ one is ultimately led to a modification of the canonical FPI algorithm in which higher order fluctuations are approximately included via the Gaussian transform effective potential of Eq. (1.12).

Figure 2 illustrates the points discussed above. Shown is a "typical" high order path (short-dashed line) constructed according to Eq. (1.11) with $100 a_{k}$ values chosen at random from a Gaussian distribution with standard deviation $\sigma_{k}(\beta=m=\hbar=1)$. Also shown (solid line) is its $k_{\max }=10$ core. In the basic FPI method one assumes that these deviations are zero, or, equivalently, that the integral

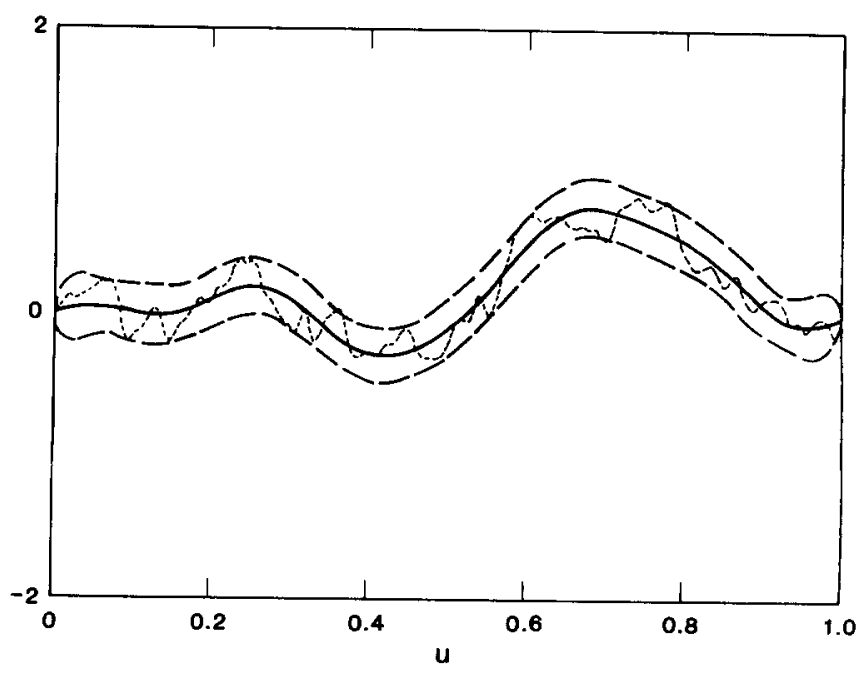

FIG. 2. Plot of $q_{10}(u)$, a "typical $k_{\text {max }}=10$ path" as discussed in text (line), "typical $k_{\max }=100$ path" built on $q_{10}(u)$ (short-dash), and partial averaging envelope function $q_{10}(u) \pm 2\left[\sigma^{2}(u)\right]^{1 / 2}$ (long-dash). 
of the potential along every high order path is equal to the corresponding integral along the low order core. The longdashed line in Fig. 2 represents a $\pm 2\left[\sigma^{2}(u)\right]^{1 / 2}$ envelope for $k_{\max }=10$ and can be seen to describe the magnitude of the high order fluctuations well. [Strictly speaking, $\sigma^{2}(u)$ is the second moment of all high order fluctuations $\left(k_{\max }+1\right.$ to $\left.\infty\right)$.]

We turn now to the question of evaluating $V_{\text {eff }}$. From Eq. (1.12) it can be seen that $V_{\text {eff }}$ is analytically attainable for any potential which is superposed from functions having analytical Gaussian transforms. Such functions include polynomials, linear exponentials, ${ }^{31}$ and Gaussians. Clearly, very flexible potential functions can be composed from these components. If $V$ is not solely comprised of Gaussian transformable functions, two procedures immediately suggest themselves.

The first of these entails approximating $V$ by Gaussian transformable functions. If the region of coordinate space accessible in the process of interest is restricted to some relatively localized region, a modest order Taylor series expansion should suffice. ${ }^{13}$ By including Gaussians and/or exponentials in the fitting procedure, asymptotic decay of interactions and steep repulsive walls can be accurately modeled. ${ }^{14}$ In this manner $V_{\text {eff }}$ can be analytically evaluated for essentially any $V$, providing sufficient care (and effort) is expended in fitting $V$ to a superposition of Gaussian transformable functions.

If this task proves too laborious, a second appealing alternative exists, namely, to perform a gradient expansion about the fluctuation variable $p$ in Eq. (1.12). Proceeding in this way, we obtain

$$
V_{\text {eff }}(q, u)=V(q)+\frac{1}{2} V^{\prime \prime}(q) \sigma^{2}(u)+\frac{1}{8} V^{(i v)}(q) \sigma^{4}(u)+\cdots .
$$

Of course, if this series is continued until it truncates (which will be at infinite order for nonpolynomial $V$ ) the exact $V_{\text {eff }}$ will be obtained. What is very appealing, however, is that in a wide variety of circumstances only the lowest order corrections will be needed. This is because $\sigma^{2}(u)$, which is a measure of the "higher-order" fluctuations, becomes uniformly smaller on $0<u<1$ as $k_{\max }$ is increased (the smaller $\beta$ is, the lower the value of $k_{\max }$ which will suffice to force the "small $\sigma^{2}$ " limit). Hence one can devise a variation of the PA prescription in which $V_{\text {eff }}$ is approximated by a low order gradient expansion [e.g., only the $V^{\prime \prime}$ correction on the right-hand side of Eq. (1.15) is kept]. The strict bound on $\rho_{\beta}$ for all $k_{\max }$ is thereby lost. However, the procedure is clearly convergent in the following sense: As $k_{\max } \rightarrow \infty$ all gradient corrections are squeezed out and the canonical FPI prescription (i.e., the exact propagator) is recovered. Moreover, as we back away from $k_{\max }=\infty$, there will be some regime where the truncated gradient expansion is identical to $V_{\text {eff }}$, i.e., where the complete PA result will be obtained. On the assumption that partial averaging improves results for all $k_{\max }$ relative to the canonical FPI procedure (an assumption which has yet to be seriously contradicted in our experience), the gradient expansion method is guaranteed to converge at lower $k_{\max }$ than its canonical FPI analog.

\section{PARTIAL AVERAGING AND GRADIENT EXPANSIONS IN MANY DIMENSIONS}

As stressed in the introductory section, the entire premise underlying the development of MCPI techniques is the ease with which they may be extended to many coupled spatial dimensions. Fortunately, the partial averaging procedure does not destroy this important feature. By manipulations entirely analogous to those discussed in the one-dimensional case, one finds for the $d$-dimensional simple Cartesian Hamiltonian

$$
\widehat{H}=\sum_{i=1}^{d} \hat{p}_{i}^{2} / 2 m_{i}+V(\hat{\mathbf{x}})
$$

the following expression:

$$
\begin{aligned}
\frac{\rho_{\beta}\left(\mathbf{x}_{f}, \mathbf{x}_{0}\right)}{\rho_{\beta}^{f p}\left(\mathbf{x}_{f}, \mathbf{x}_{0}\right)} \geqslant & {\left[\prod_{i=1}^{d}\left\{\prod_{k=1}^{k_{i}} 2 \pi \sigma_{k}^{2}\right\}\right]^{-1 / 2} } \\
& \times \prod_{i=1}^{d}\left\{\int d a_{1}^{(i)} \ldots d a_{k_{i}}^{(i)} w\left(\mathbf{a}^{(i)}\right)\right\} \\
& \times \exp \left[-\beta \int_{0}^{1} d u V_{\text {eff }}\left(\mathbf{x}_{k}(u), u\right)\right],
\end{aligned}
$$

where the following notation has been adopted: $w\left(\mathbf{a}^{(i)}\right)$ is the Gaussian weight factor for the Fourier coefficients associated with the $i$ th spatial coordinate, $x^{(i)}$ :

$$
w\left(\mathbf{a}^{(i)}\right)=\exp \left\{-\sum_{k=1}^{k_{i}} a_{k}^{(i) 2} / 2 \sigma_{k}^{2}\right\} .
$$

[The fluctuation parameters $\sigma_{k}^{2}$ were given in Eq. (1.5)]. $k_{i}$ corresponds to the maximum Fourier coefficient utilized to describe the Fourier path associated with $x^{(i)}$. In other words, $\mathbf{x}_{k}(u) \equiv\left(x_{k_{1}}^{(1)}(u), \ldots, x_{k_{d}}^{(d)}(u)\right)$, with

$$
x_{k_{i}}^{(i)}(u)=x_{0}^{(i)}+\left(x_{f}^{(i)}-x_{0}^{(i)}\right) u+\sum_{k=1}^{k_{i}} a_{k}^{(i)} \sin (k \pi u) .
$$

The critical specification concerns the definition of $V_{\text {eff }}$. Again, this definition is completely analogous to the one put forth in the $1 \mathrm{~d}$ case. To make it explicit let us notate $V$ by components, i.e., $V(\mathbf{x}) \rightarrow V\left(x^{(1)}, \ldots, x^{(d)}\right)$. Then

$$
\begin{aligned}
V_{\text {eff }}(\mathbf{x}, u)= & {\left[\prod_{i=1}^{d} 2 \pi \sigma_{i}^{2}(u)\right]^{-1 / 2} \int d p_{1} \ldots d p_{d} } \\
& \times \exp \left[-\sum_{i=1}^{d} p_{i}^{2} / 2 \sigma_{i}^{2}(u)\right] \\
& \times V\left(x^{(1)}+p_{1}, \ldots, x^{(d)}+p_{d}\right)
\end{aligned}
$$

with $\sigma_{i}^{2}(u)$ analogous to the $1 \mathrm{~d}$ case:

$$
\begin{aligned}
\sigma_{i}^{2}(u) & =\sum_{k=k_{i}+1}^{\infty} \sigma_{k}^{2} \sin (k \pi u) \\
& =\frac{\hbar^{2} \beta}{m} u(1-u)-\sum_{k=1}^{k_{i}} \sigma_{k}^{2} \sin ^{2}(k \pi u) \\
& =\frac{\hbar^{2} \beta}{m}\left[u(1-u)-\frac{2}{\pi^{2}} \sum_{k=1}^{k_{i}} \frac{\sin ^{2}(k \pi u)}{k^{2}}\right] .
\end{aligned}
$$

Thus we see that the effective potential relevant to multidimensional Hamiltonians is a $d$-dimensional Gaussian transform of the system potential $V(\mathbf{x})$. Note that for a separable potential $V(\mathbf{x})=\Sigma_{i=1}^{d} v\left(x^{(d)}\right)$, the effective potential 
breaks up into a sum of $1 \mathrm{~d}$ effective potentials. This is obviously the correct reduction of Eq. (2.2) in the separable potential limit. Also analogously to the 1d case, potentials composed of polynomials, linear exponentials, and Gaussians (including Gaussians with quadratic cross coupling in the exponent) may be transformed analytically.

Finally, the gradient expansion procedure discussed above in connection with the $1 \mathrm{~d}$ problem generalizes easily to the multidimensional case. Indeed, because of the Gaussian transform structure of Eq. (2.2), many terms in the Taylor series expansion of $V$ about $p_{1}, \ldots, p_{d}=0$ vanish. Through fourth order we find

$$
\begin{aligned}
V_{\text {eff }}(\mathbf{x}, u)= & V(\mathbf{x})+\frac{1}{2} \sum_{j=1}^{d} V_{f} \sigma_{j}^{2}(u)+\frac{1}{8} \sum_{j=1}^{d} V_{f} \sigma_{j}^{4}(u) \\
& +\frac{1}{4} \sum_{j>k} V_{j^{2} k^{2}} \sigma_{j}^{2}(u) \sigma_{k}^{2}(u)+\cdots
\end{aligned}
$$

In Eq. (2.3), $V_{f^{2}}$, etc., are derivatives of the potential evaluated at $\mathrm{x}$, e.g., $V_{f^{2} k^{3}}=\partial_{x^{(j)}}^{2} \partial_{x^{(k)}}^{3} V(\mathrm{x})$. The lowest neglected terms are of overall order $\sigma^{6}$.

\section{CUMULANT APPROACH TO PARTIAL AVERAGING}

In discussing partial averaging in imaginary time we introduced the only approximation involved via Gibbs' inequality ${ }^{15}$ :

$$
\langle\exp \{f(\mathbf{x})\}\rangle \geqslant \exp \{\langle f(\mathbf{x})\rangle\} .
$$

This inequality is quite general (it applies for arbitrary probability distributions in configuration spaces of arbitrary dimensionality). By applying it to the problem of interest in Secs. I and II it was possible to demonstrate that the PA prescription approximation to $\rho_{\beta}\left(\mathbf{x}_{f}, \mathbf{x}_{0}\right)$ [Eq. (1.4) in 1d, and Eq. (2.1) ind dimensions] rigorously bounded the exact value from below for any $\mathbf{x}_{0}, \mathbf{x}_{f}$.

It is also possible to look at the right-hand side of Eq. (3.1) as the first term in a cumulant expansion ${ }^{16}$ :

$$
\begin{aligned}
\int d \mathbf{x} Q(\mathbf{x}) e^{f(\mathbf{x})}= & \exp \left\{\bar{f}+\frac{1}{2}\left(\overline{f^{2}}-\bar{f}^{2}\right)\right. \\
& \left.+\frac{1}{3 !}\left(\overline{f^{3}}-3 \overline{f^{2}} \bar{f}+2 \bar{f}^{3}\right)+\cdots\right\},
\end{aligned}
$$

where $\bar{g} \equiv \int d \mathbf{x} Q(\mathbf{x}) g(\mathbf{x})$. It is important to note that $Q(\mathbf{x})$ does not have to be positive definite or even real for Eq. (3.2) to hold.

There are both advantages and disadvantages to viewing the PA prescription as a consequence of Eq. (3.2) rather than Eq. (3.1). The only disadvantage is that for problems set in imaginary time, the rigorous bound on $\rho_{\beta}\left(\mathbf{x}_{f}, \mathbf{x}_{0}\right)$ is lost if the expansion on the right-hand side of Eq. (3.2) is pursued beyond the first term. In practice, however, we have not yet been able to exploit the "lower bound" property of the PA prescription in a practical way. There are at least two reasons for this. First, formal bounds will not necessarily be respected when an integral is computed by importance sampling, due to the error bars inherent in results obtained by MC simulations. And second, the only obvious tractable zeroth order problem which has parameters amenable to variational optimization is the harmonic oscillator problem. It has been our experience that highly anharmonic (e.g., double well potential) results are relatively insensitive to adjustments in the frequencies of harmonic oscillator reference systems. ${ }^{17}$ Finally, even if one can use the variational features of PA in imaginary time to advantage, many problems of considerable interest involve "complex time" dynamics, i.e., $\beta \rightarrow \beta+i t / \hbar$ in the expressions used in the development presented up to this point. (Derivation of complex time expressions will be given in the next section.) Then Gibbs' inequality breaks down, because the "weight function" $Q(\mathbf{x})$ in Eq. (3.2) becomes complex. However, the cumulant expansion given on the right-hand side of Eq. (3.2) remains valid and indeed enables implementation of PA when temporal evolution $(t \neq 0)$ is required.

We now discuss the advantages of a cumulant approach to PA. One of these has just been mentioned: All manipulations used in obtaining the PA prescription for imaginary time propagators follow analogously in the complex time case if the approximation of replacing the "average of the exponential" by the "exponential of the average" is viewed as a first order cumulant expansion procedure. Another advantage is the utility of the cumulant expansion for deriving power series expansions in small parameters of interest. $\mathrm{Ob}$ vious choices of this small parameter include $\hbar$ (which results in Wigner-Kirkwood expansions of the density opera$\operatorname{tor}^{18}$ ) and $\beta$ (which results in improved small $\beta$ propagators).

A third potential advantage of the cumulant approach revolves around the possibility of improving the convergence rate of MCPI simulations by explicitly computing higher cumulants on the right-hand side of Eq. (3.2). For example, it is straightforward to derive an implementable second cumulant correction to the first order result $-\beta \int_{0}^{1} d u V_{\text {eff }}(\mathbf{x}(u), u)$ of Eq. (2.1). For simplicity we will discuss the correction in one spatial dimension. Generalization to $d$ dimensions should be obvious.

Appealing to Eq. (3.2) one finds [cf. Eq. (1.6)]

$$
\begin{aligned}
& \left\langle\exp \left[-\beta \int_{0}^{1} d u V(q(u))\right]\right\rangle \\
& \cong \exp \left\{-\beta\left\langle\int_{0}^{1} d u V(q(u))\right\rangle+\frac{\beta^{2}}{2}\left(\left\langle\left[\int_{0}^{1} d u V(q(u))\right]^{2}\right\rangle-\left\langle\int_{0}^{1} d u V(q(u))\right\rangle^{2}\right)\right\} \\
& \quad=\exp \left\{-\beta \int_{0}^{1} d u V_{\text {eff }}\left(q_{k_{\max }}(u), u\right)+\frac{\beta^{2}}{2}\left(\int_{0}^{1} d u \int_{0}^{1} d u^{\prime} G\left(u, u^{\prime}\right)-\left[\int_{0}^{1} d u V_{\text {eff }}\left(q_{k_{\max }}(u), u\right)\right]^{2}\right)\right\}
\end{aligned}
$$


The function $G\left(u, u^{\prime}\right)$ will be prescribed momentarily. First a few notational clarifications are indicted. Angled brackets here indicate Gaussian averages over the Fourier variables $a_{k_{\max }+1}, \ldots, a_{\infty}$. In other words, what was notated as ( $)_{a_{k_{\max }+1, \ldots, a_{\infty}}}$ in Eqs. (1.6)-(1.8) is now designated simply by \langle\rangle . Furthermore, $q(u)$ is the infinite coefficient Fourier path given in Eq. (1.4), while $q_{k_{\max }}(u)$ is the truncated path of Eq. (1.11). Finally, we turn to the specification of $G$ :

$$
\begin{aligned}
& G\left(u, u^{\prime}\right)=\left\langle V(q(u)) V\left(q\left(u^{\prime}\right)\right)\right\rangle \\
& =\left[(2 \pi)^{2} \operatorname{det} \mathrm{A}\right]^{-1 / 2} \int d p_{1} d p_{2} \\
& \times \exp \left[-\frac{1}{2} \mathbf{p} \cdot \mathrm{A}^{-1} \cdot \mathbf{p}\right] V\left(q_{k_{\max }}(u)+p_{1}\right) \\
& \times V\left(q_{k_{\max }}\left(u^{\prime}\right)+p_{2}\right),
\end{aligned}
$$

where $\mathbf{p}=\left(p_{1}, p_{2}\right)$ and the components of $\mathrm{A}$ are

$$
\begin{gathered}
A_{11}=\sum_{k=k_{\max }+1}^{\infty} \sigma_{k}^{2} \sin ^{2}(k \pi u) ; \\
A_{22}=\sum_{k=k_{\max }+1}^{\infty} \sigma_{k}^{2} \sin ^{2}(k \pi u), \\
A_{12}=A_{21}=\sum_{k=k_{\max }+1}^{\infty} \sigma_{k}^{2} \sin (k \pi u) \sin \left(k \pi u^{\prime}\right) \\
=\frac{\hbar^{2} \beta}{m} u_{1}\left(1-u_{g}\right)-\sum_{k=1}^{k_{\max }} \sigma_{k}^{2} \\
\quad \times \sin (k \pi u) \sin \left(k \pi u^{\prime}\right) .
\end{gathered}
$$

The last line of Eq. (3.5) shows how the evaluation of $A_{i j}$ can be simplified. The symbol $u_{g(1)}$ stands for the greater (lesser) value of $u, u^{\prime}$.

\section{PARTIAL AVERAGING IN COMPLEX TIME}

In this section we wish to consider the application of the partial averaging prescription to the problem of computing $\rho_{\beta_{c}}\left(\mathbf{x}_{f}, \mathbf{x}_{0}\right)$ when $\beta_{c}=\beta+i t / \hbar$. The relevant results are easy to present because they are identical in form to the imaginary time analogs discussed in previous sections upon replacement of $\beta \rightarrow \beta_{c}$. More specifically, the Fourier coefficient path integral for $\rho_{\beta_{c}}$ is

$$
\begin{aligned}
\frac{\rho_{\beta_{c}}\left(x_{f}, x_{0}\right)}{\rho_{\beta_{c}}^{f}\left(x_{f}, x_{0}\right)}= & {\left[\prod_{k=1}^{\infty} 2 \pi \tilde{\sigma}_{k}^{2}\right]^{-1 / 2} \int d a_{1} \ldots d a_{\infty} } \\
& \times \exp -\sum_{k=1}^{\infty} a_{k}^{2} / 2 \tilde{\sigma}_{k}^{2}-\beta_{c} \int_{0}^{1} d u V(q(u))
\end{aligned}
$$

with

$$
\tilde{\sigma}_{k}^{2}=2 \hbar^{2} \beta_{c} / m(\pi k)^{2},
$$

$q(u)$ the generic path of Eq. (1.4), and $\rho_{\beta_{c}}^{f p}$ the free-particle propagator for complex $\beta_{c}$ [Eq. (1.3) with $\beta \rightarrow \beta_{c}$ ]. Two remarks are appropriate here: (1) For notational simplicity, only the 1d case will be discussed here. The multi- $d$ generalizations are obvious. (2) Since little attention has been devoted to complex time Fourier coefficient path integration in previous literature, a derivation of Eq. (4.1) is given in the
Appendix. With these remarks completed, we can proceed to partially average the formally exact complex time Fourier path integral.

Starting from Eq. (4.1), the integrals over $a_{k_{\max }+1}, \ldots, a_{\infty}$ may be approximated by the first term in a cumulant expansion in order to obtain

$$
\begin{aligned}
\frac{\rho_{\beta_{c}}\left(x_{f}, x_{0}\right)}{\rho_{\beta_{c}}^{f p}\left(x_{f}, x_{0}\right)} \cong & {\left[\prod_{k=1}^{k_{\max }} 2 \pi \tilde{\sigma}_{k}^{2}\right]^{-1 / 2} } \\
& \times \int d a_{1} \ldots d a_{k_{\max }} \\
& \times \exp \left[-\sum_{k=1}^{k_{\max }} a_{k}^{2} / 2 \tilde{\sigma}_{k}^{2}\right. \\
& \left.-\beta_{c} \int_{0}^{1} d u V_{\text {eff }}\left(q_{k_{\max }}(u), u\right)\right],
\end{aligned}
$$

with $q_{k_{\max }}(u)$ the low order Fourier path of Eq. (1.11), and $V_{\text {eff }}$ having the same structure as the imaginary time cases [cf. Eq. (1.13) ], except that $\beta \rightarrow \beta_{c}, \sigma_{k} \rightarrow \tilde{\sigma}_{k}$. Higher cumulants follow in a similar fashion.

\section{IMPROVED SMALL ARGUMENT PROPAGATORS}

Suppose that we wish to improve upon the basic small argument propagator

$$
\begin{aligned}
\rho_{\epsilon}\left(x_{f}, x_{0}\right) & \equiv\left\langle x_{f}\left|e^{-\epsilon \hat{H}}\right| x_{0}\right\rangle \\
& =\rho_{\epsilon \rightarrow 0}^{f p}\left(x_{f}, x_{0}\right) \exp \left\{-\frac{\epsilon}{2}\left[V\left(x_{f}\right)+V\left(x_{0}\right)\right]\right\},
\end{aligned}
$$

$\rho_{\epsilon}^{f p}$ being the free-particle propagator of Eq. (1.3) with $\beta \rightarrow \epsilon$. One would hope to be able to find an effective potential $\bar{V}\left(x_{f}, x_{0}\right)$, which is both easy to evaluate and improves the accuracy of the right-hand side of Eq. (5.1) when it is substituted for $\left[V\left(x_{f}\right)+V\left(x_{0}\right)\right] / 2$ in that expression. Such a function can in fact be obtained using the PA prescription.

Starting from the formally exact FPI expression, Eq. (1.2), and applying Gibbs' inequality to all the Fourier coefficients, one obtains

$$
\rho_{\beta}\left(x_{f}, x_{0}\right) \geqslant \rho_{\beta}^{f p}\left(x_{f}, x_{0}\right) \exp \left[-\beta \int_{0}^{1} d u V_{\text {eff }}(u)\right],
$$

where

$$
\begin{aligned}
V_{\text {eff }}(u)= & {\left[2 \pi \sigma^{2}(u)\right]^{-1 / 2} } \\
& \times \int_{-\infty}^{\infty} e^{-p^{2} / 2 \sigma^{2}(u)} V\left(x_{0}+\left(x_{f}-x_{0}\right) u+p\right)
\end{aligned}
$$

and

$$
\sigma^{2}(u)=\frac{\beta \hbar^{2}}{m} u(1-u) .
$$

The inequality, Eq. (5.2) holds for all values of $\beta$. However, for sufficiently small $\beta$ this inequality becomes more and more sharply peaked $\left[\sigma^{2}(u)\right.$ in Eq. (5.4) becomes uniformly smaller]. Comparing Eq. (5.2) to Eq. (5.1) in the limit of $\beta \rightarrow \epsilon$ small, one is naturally tempted to identify

$$
\bar{V}\left(x_{f}, x_{0}\right)=\int_{0}^{1} d u V_{\text {eff }}(u),
$$

where $V_{\text {eff }}(u)$ was given in Eq. (5.3), and $\sigma^{2}(u)$ in Eq. (5.4) 
(with $\beta \rightarrow \epsilon$ there). In other words, the Gaussian transformed potential Eq. (5.3), when averaged (integrated) over $u$ is the improved small argument potential which replaces $\left[V\left(x_{f}\right)+V\left(x_{0}\right)\right] / 2$ in the small argument propagator, Eq. (5.1). In the next section we will explicitly demonstrate that $\bar{V}\left(x_{f}, x_{0}\right)$ in Eq. (5.5) is an improvement over $\left[V\left(x_{f}\right)+V\left(x_{0}\right)\right] / 2$, by showing that the $\bar{V}\left(x_{f}, x_{0}\right)$ small argument propagator is accurate through $O\left(\epsilon^{2}\right)$, whereas the version in Eq. (5.1) is accurate only through $O(\epsilon)$. In closing this section we note that the function $\bar{V}$ has been identified in a slightly different but closely related context by Schweizer et al. ${ }^{6}$ [cf. their Eqs. (2.14) and (2.15) with the (free particle) choice $\rho_{0}$, as given in their Eq. (3.4); note that their $\beta / P$ is our $\epsilon]$ via a considerably different method than the one employed here. They were pursuing an upper bound on the thermodynamic free energy. It is easy to see how such a bound can be deduced through the derivation pursued above: By composing finite argument propagators out of small argument approximations $\rho_{\epsilon}^{f p}\left(x_{f}, x_{0}\right)$ $\times \exp \left[-\epsilon \bar{V}\left(x_{f}, x_{0}\right)\right] \leqslant \rho_{\epsilon}\left(x_{f}, x_{0}\right)$, the approximation to the finite argument propagator which is thereby obtained must itself be a lower bound on $\rho_{\beta}\left(x_{f}, x_{0}\right)$. Hence the partition function obtained from such a composition must also be a lower bound on the exact result.

\section{EXACT $O\left(\epsilon^{2}\right)$ PROPAGATOR FROM PARTIAL AVERAGING}

From Sec. III we know that Eq. (5.2) can be viewed as the simplest approximation to the formally exact expression

$$
\begin{aligned}
\rho_{\beta}\left(x_{f}, x_{0}\right)= & \rho_{\beta}^{f p}\left(x_{f}, x_{0}\right) \\
& \times \exp \left[-\beta \int_{0}^{1} d u V_{\mathrm{eff}}(u) d u\right. \\
& +\frac{\beta^{2}}{2}\left(\left\langle\left[\int_{0}^{1} d u V(q(u)]^{2}\right\rangle\right.\right. \\
& \left.\left.-\left\langle\int_{0}^{1} d u V(q(u))\right\rangle^{2}\right)+O\left(\beta^{3}\right)\right] .
\end{aligned}
$$

Here we are adapting Eq. (3.3a) to our present needs. $V_{\text {eff }}(u)$ was given in Eq. (5.3). $q(u)$ is the full Fourier path of Eq. (1.4), and the angled brackets indicate the appropriate Gaussian averages over all Fourier coefficients. Clearly, as $\beta \rightarrow 0$ all the relevant Gaussian distributions become very sharply peaked around 0 , so that the effect of \langle\rangle in this limit is simply to zero all Fourier coefficients encompassed. That is,

$$
\begin{aligned}
& \left\langle\left[\int_{0}^{1} d u V(q(u))\right]^{2}\right\rangle-\left\langle\int_{0}^{1} d u V(q(u))\right\rangle^{2} \\
& \quad=c_{1} \beta+c_{2} \beta^{2}+\cdots
\end{aligned}
$$

with the zeroth order coefficient $c_{0}$ conspicuously absent. ${ }^{19}$ Thus, it must be the case that truncation of Eq. (6.1) after the first cumulant [i.e., Eq. (5.2)] is accurate through order $\beta^{2}$. We can explicitly verify this in the following way.

Let us consider $\beta$ to be a small number $\epsilon$ and extract the part of $\epsilon \int_{0}^{1} d u V_{\text {eff }}(u)$ which contributes to $O\left(\epsilon^{2}\right)$. Essentially we will expand in Taylor series and disgard terms of $O\left(\epsilon^{3}\right)$ and higher. One subtle point in our power counting procedure is that we will regard the quantity $\left(x_{f}-x_{0}\right)$ as $O\left(\epsilon^{1 / 2}\right)$.
Naively, this is because the kinetic energy factor, $\exp \left\{-m\left(x_{f}-x_{0}\right)^{2} / 2 \hbar^{2} \epsilon\right\}$ in $\rho^{f p}$, effectively restricts $\left(x_{f}-x_{0}\right) \leqslant \epsilon^{1 / 2}$. A more precise justification of this scheme will be given after we have obtained the desired result.

In order to evaluate the propagator to $O\left(\epsilon^{2}\right)$ we need to evaluate $\int_{0}^{1} d u V_{\text {eff }}(u)$ to $O(\epsilon)$. The place to begin, therefore, is by expanding $V_{\text {eff }}(u)$ :

$$
\begin{aligned}
V_{\text {eff }}(u)= & V\left(x_{0}+\left(x_{f}-x_{0}\right) u\right) \\
& +\frac{1}{2} V^{\prime \prime}\left(x_{0}+\left(x_{f}-x_{0}\right) u\right) \sigma^{2}(u)+\cdots .
\end{aligned}
$$

There is no need to continue the series further because $\sigma^{2}(u) \sim \epsilon$, and according to the gradient expansion procedure developed in Sec. I [cf. Eq. (1.15)] the next term $\sim \sigma^{4} \sim \epsilon^{2}$. Next we expand $V\left[x_{0}+\left(x_{f}-x_{0}\right) u\right]$ through order $\left(x_{f}-x_{0}\right)^{2} \sim \epsilon$. At the same time we can replace $V^{\prime \prime}\left(x_{0}+\left(x_{f}-x_{0}\right) u\right) \rightarrow V^{\prime \prime}\left(x_{0}\right)$ since $V^{\prime \prime}$ is multiplied by a term $\left(\sigma^{2}\right)$ of order $\epsilon$. Upon integrating the resultant approximation to $V_{\text {eff }}$, we find

$$
\begin{aligned}
\int_{0}^{1} d u V_{\mathrm{eff}}(u)= & V\left(x_{0}\right)+V^{\prime}\left(x_{0}\right)\left(x_{f}-x_{0}\right) / 2 \\
& +V^{\prime \prime}\left(x_{0}\right)\left(x_{f}-x_{0}\right)^{2} / 6 \\
& +V^{\prime \prime}\left(x_{0}\right) \hbar^{2} \epsilon / 12 m+O\left(\epsilon^{2}\right) \\
= & \widetilde{V}\left(x_{f}, x_{0}\right)+O\left(\epsilon^{2}\right)
\end{aligned}
$$

where

$$
\begin{aligned}
\widetilde{V}\left(x_{f}, x_{0}\right) \equiv & \frac{1}{2}\left[V\left(x_{0}\right)+V\left(x_{f}\right)\right] \\
& +\frac{V^{\prime \prime}}{12}\left[\frac{\hbar^{2} \epsilon}{m}-\left(x_{f}-x_{0}\right)^{2}\right] .
\end{aligned}
$$

Equation (6.4b), which is our final answer, was obtained via obvious Taylor series manipulations from Eq. (6.4a). Moreover, the coefficient $V^{\prime \prime}$ appearing in Eq. (6.4c) has been vaguely notated on purpose, since the number which is set here is not unique. Natural choices are $V^{\prime \prime}=a V^{\prime \prime}\left(x_{0}\right)+b V^{\prime \prime}\left(x_{f}\right) \quad$ with $a+b=1 \quad$ and $V^{\prime \prime}=V^{\prime \prime}\left(\left(x_{0}+x_{f}\right) / 2\right)$.

The discussion of the previous paragraph has thus resulted in the explicit improved small argument propagator

$\rho_{\epsilon}\left(x_{f}, x_{0}\right)=\rho_{\epsilon}^{f p}\left(x_{f}, x_{0}\right) \exp \left[-\epsilon \widetilde{V}\left(x_{f}-x_{0}\right)\right]+O\left(\epsilon^{2}\right)$.

We will now indicate precisely in what sense this expression is correct through $O\left(\epsilon^{2}\right)$. [This will serve to justify counting $\left(x_{f}-x_{0}\right)$ as $O\left(\epsilon^{1 / 2}\right)$ in the derivation.]

Consider the evolution of an initially localized wave packet $\psi(x, 0)$ under the imaginary time Schrödinger equation:

$$
-\partial_{t} \psi(x, t)=h_{x} \psi(x, t),
$$

$h_{x}$ being the coordinate space representation of $\hat{H}^{20}$ The exact propagator $\rho_{\epsilon}$ is defined so as to have the property

$$
\begin{aligned}
\psi(x, \epsilon) & =\sum_{k=1}^{\infty} \frac{\left(-\epsilon h_{x}\right)^{k}}{k !} \psi(x, 0) \\
& =\int d x_{0} \rho_{\epsilon}\left(x, x_{0}\right) \psi\left(x_{0}, 0\right) .
\end{aligned}
$$

Feynman ${ }^{1}$ demonstrated that the small argument propaga- 
tor function given in Eq. (5.1) is "correct to first order in $\epsilon$ " by inserting it into Eq. (6.7b) and expanding in a Taylor series about $\epsilon$. He found that the right-hand side of Eq. (6.7a) was recovered through $O(\epsilon)$. If the analogous calculation is performed using the improved small argument propagator in Eq. (6.5), one finds that the right-hand side of Eq. (6.7a) is recovered through $O\left(\epsilon^{2}\right)$.

In concluding this section we note that for $V(x)=m \omega^{2} x^{2} / 2, \bar{V}\left(x_{f}, x_{0}\right)=\widetilde{V}\left(x_{f}, x_{0}\right)$ exactly and with no ambiguities $\left(V^{\prime \prime}=\right.$ constant $\left.=m \omega^{2}\right)$. Thus our general expression for $\widetilde{V}$ in Eq. (6.4c) reduces to the result for $\bar{V}$ given by Schweizer et al. ${ }^{6}$ in their discussion of harmonic oscillator dynamics [cf. their Eq. (3.9)]. For the general case of motion in anharmonic potentials Thirumalai and Berne $^{5}$ have presented an improved propagator result similar to the one given in Eq. (6.4b). However, their result differs from ours in the details of the second derivative terms which constitute the $O\left(\epsilon^{2}\right)$ correction.

\section{PARTIAL AVERAGING APPLIED TO HARMONIC OSCILLATOR SYSTEMS}

In this section we wish to examine various examples of motion in a ld harmonic oscillator well. It will prove convenient to organize the discussion so that imaginary time behavior is considered first, then complex time behavior. However, the essential feature of PA in harmonic oscillator problems is the same in both imaginary and complex time cases: The propagator obtained in the canonical FPI procedure is rescaled by a simple factor which tends to zero in the limit that $k_{\max } \rightarrow \infty$. Let us first derive this correction factor, and then see how it affects the calculation of various properties.

Consider the complex time propagator $\rho_{\beta_{c}}\left(x_{f}, x_{0}\right)$ for a harmonic oscillator potential $V(x)=m \omega^{2} x^{2} / 2$. According to the PA prescription the appropriate effective potential which should be utilized in a FPI computation involving $k_{\max }$ coefficients is

$$
V_{\text {eff }}\left(q_{k_{\max }}(u), u\right)=\frac{m \omega^{2}}{2}\left[g_{k_{\max }}(u)^{2}+\tilde{\sigma}^{2}(u)\right],
$$

where $q_{k_{\max }}$ is a Fourier path composed of the lowest $k_{\max }$ sine waves [Eq. (1.11)] and $\tilde{\sigma}^{2}(u)$ was given in Eq. (4.2). It is easy to evaluate

$$
\begin{aligned}
\beta_{c} \int_{0}^{1} d u V_{\text {eff }}\left(q_{k_{\max }}(u), u\right) \\
=\frac{\beta_{c} m \omega^{2}}{2} \int_{0}^{1} d u q_{k_{\max }}(u)^{2} \\
\quad+\frac{\left(\beta_{c} \hbar \omega\right)^{2}}{12}\left[1-\frac{6}{\pi^{2}} \sum_{k=1}^{k_{\max }} \frac{1}{k^{2}}\right] .
\end{aligned}
$$

Thus, the net modification of the canonical FPI prescription induced by partial averaging is an overall scale factor, as mentioned above. Specifically,

$$
\begin{aligned}
\rho_{\beta_{c}}^{\mathrm{PA}}\left(x_{f}, x_{0}\right)= & \rho_{\beta_{\mathrm{c}}}^{\mathrm{FI}}\left(x_{f}, x_{0}\right) \\
& \times \exp \left\{-\frac{\left(\beta_{c} \hbar \omega\right)^{2}}{12}\left[1-\frac{6}{\pi^{2}} \sum_{k=1}^{k_{\max }} \frac{1}{k^{2}}\right]\right\} .
\end{aligned}
$$

In Eq. (7.1) we have superscripted the PA result to distinguish it from the analogous canonical FPI result. For completeness we record this latter function:

$$
\begin{aligned}
\rho_{B_{c}}^{\mathrm{FPI}}\left(x_{f}, x_{0}\right)= & \rho_{\beta_{c}}^{f_{c}}\left(x_{f}, x_{0}\right)\left[\prod_{k=1}^{k_{\max }} 1+(\gamma / k)^{2}\right]^{-1 / 2} \\
& \times \exp \left\{\frac{-m \omega^{2} \beta_{c}}{6}\left[x_{0}^{2}+x_{0} x_{f}+x_{f}^{2}\right]\right. \\
& \left.+\frac{m \omega^{2} \beta_{c} \gamma^{2}}{\pi^{2}} \sum_{k=1}^{k_{\max }}\left(x_{0}-(-1)^{k} x_{f}\right)^{2} g_{k}\right\}
\end{aligned}
$$

with $g_{k} \equiv\left[k^{2}\left(k^{2}+\gamma^{2}\right)\right]^{-1}$ and $\gamma$ the dimensionless constant $\gamma=\beta_{c} \hbar \omega / \pi$. Let us now proceed to utilize Eqs. (7.1) and (7.2) in calculating a number of observable properties. As indicated at the outset of the section, imaginary time properties will be considered first, and complex time properties subsequently.

The imaginary time density propagator or density matrix $\rho_{\beta}\left(x_{f}, x_{0}\right)$, contains all thermodynamic information about the system governed by a given Hamiltonian, since knowledge of $\rho_{\beta}$ enables one to compute the partition func$\operatorname{tion} Z_{\beta}=\operatorname{tr} \hat{\rho}_{\beta} \cdot \rho_{\beta}$ also contains all position and momentum density information about the system, so that various static averages,

$$
\langle F(\hat{p}, \hat{x})\rangle \equiv \operatorname{tr} \hat{\rho}_{\beta} F(\hat{p}, \hat{x}) / \operatorname{tr} \hat{\rho}_{\beta}
$$

can also be computed once $\rho_{\beta}$ has been obtained. From Eq. (7.1) it is clear that such averages will not be changed by implementation of PA, since the only difference between canonical and partially averaged versions is a $c$-number factor which cancels out of the ratios involved in Eq. (7.3). On the other hand, the partition function will change: it will be lowered by the exponential factor indicated in Eq. (7.1). To demonstrate the effect of this correction on the accuracy of $Z_{\beta}$, calculations based on a number of approximations to $Z_{\beta}$ are compared to the exact value $Z_{\beta}=\frac{1}{2} \sinh (\beta \hbar \omega / 2)$ over a considerable range of $\beta \hbar \omega$. This is done in Fig. 3 for $k_{\max }$ $=10$. Again, for completeness we exhibit the formula for the FPI approximation, which follows trivially from Eq. (7.2):

$$
Z_{\beta}^{\mathrm{FPI}}=\left\{(\beta \hbar \omega)^{2}\left[\prod_{k=1}^{k_{\max }} 1+(\gamma / k)^{2}\right]\left(1-G_{k_{\max }}\right)\right\}^{-1 / 2}
$$

with

$$
G_{k_{\max }}=\frac{2 \gamma^{2}}{\pi^{2}} \sum_{k=1}^{k_{\max }}\left(1-(-1)^{k}\right)^{2} g_{k},
$$

and in accordance with our previous notation $\gamma=\beta \hbar \omega / \pi$. The reduction factor appropriate to the PA result has been given above. Finally, the discretized Cartesian PI result (based on a trapezoid rule short time propagator) is given by $^{6}$

$$
Z_{\beta}^{D}=f^{P / 2} /\left(f^{P}-1\right)
$$

with $f=1+R^{2}+\frac{1}{2} R\left(4+R^{2}\right)^{1 / 2}, \quad R=\beta \hbar \omega / P$, $P=k_{\max }+1$. The PA result is indistinguishable at this level of resolution from the exact result over the range of $\beta \hbar \omega$ plotted in Fig. 3. Examination under higher resolution re- 


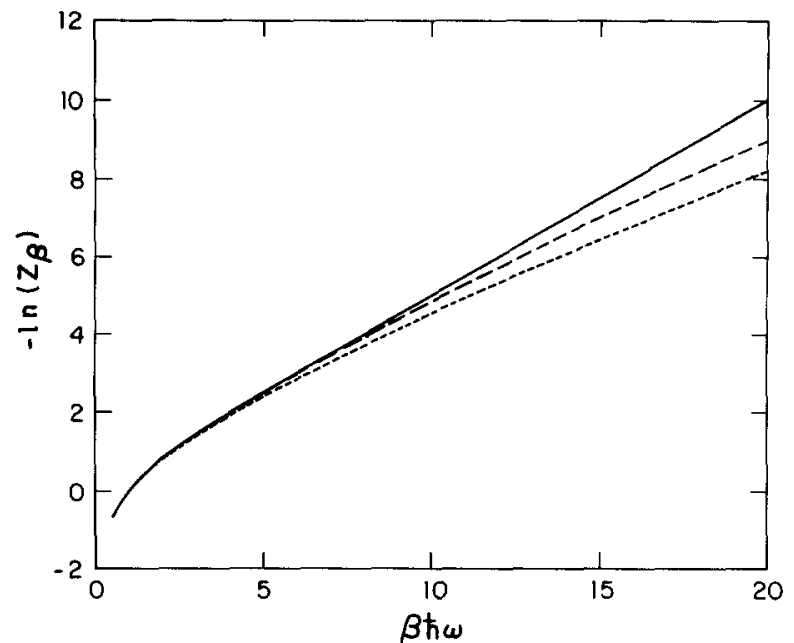

FIG. 3. $-\ln Z_{\beta}$ vs $\beta \hbar \omega$ for the harmonic oscillator system described in Sec. VII. The exact result (solid line), which is indistinguishable from the PA-FPI result, is compared with $k_{\max }=10=P$ canonical FPI (shortdash), and trapezoid (long-dash) approximations.

veals that the PA solution provides a strict lower bound on $Z_{\beta}$, as discussed in Sec. I. The improvement of the PA result relative to its canonical FPI analog is striking: Without PA, the Fourier prescription converges noticeably slower than the $P$ discretized results of Schweizer $e t$ al. The PA corrected result, on the other hand, converges noticeably faster.

As has been remarked above, for a harmonic oscillator system partial averaging does not alter static averages $\langle F(\hat{p}, \hat{x})\rangle$ relative to the canonical FPI prediction, because there is no coordinate dependence in the PA correction to $\rho_{\beta}\left(x_{f}, x_{0}\right)$. In view of the dramatic improvement obtained in $Z_{\beta}$ upon application of PA, it might be expected that quantities such as $\left\langle\hat{x}^{2}\right\rangle,\left\langle p^{2}\right\rangle$ are predicted rather accurately by the canonical FPI prescription, since they are not improved by PA. More precisely, they are not improved by the first cumulant correction in the PA formalism. They will be modified at second order, as we will see below. However, it is instructive at this point to examine the average potential and kinetic energies for some of the simpler approximations as a function of $\beta \hbar \omega$. These are to be compared to the exact result $\langle P E\rangle / \hbar \omega=\langle K E\rangle / \hbar \omega=\operatorname{coth}(\beta \hbar \omega / 2) / 4$. For the FPI procedure (with or without first cumulant PA), we find from Eq. (7.1) that

$$
\begin{aligned}
& \frac{1}{\hbar \omega} \cdot m \omega^{2}\left\langle\hat{x}^{2}\right\rangle / 2 \\
& =\left\{2 \beta \hbar \omega\left(1-\frac{2 \gamma^{2}}{\pi^{2}} \sum_{k=1}^{k_{\max }}\left[1-(-1)^{k}\right]^{2} g_{k}\right\}^{-1},\right. \\
& \frac{1}{\hbar \omega} \cdot\left\langle\hat{p}^{2}\right\rangle / 2 m \\
& =\frac{\beta \hbar \omega}{2}\left\{1 /(\beta \hbar \omega)^{2}+\frac{1}{12}-\frac{2(\beta \hbar \omega)^{2}}{\pi^{4}}\right. \\
& \left.\quad \times \sum_{k=1}^{k_{\max }} g_{k}+\frac{(\beta \hbar \omega)^{2}}{2 \pi^{4}} \sum_{k=1}^{k_{\max }}\left[1-(-1)^{k}\right]^{2} g_{k}\right] .
\end{aligned}
$$

The analogous expressions in the discretized Cartesian PI prescription are

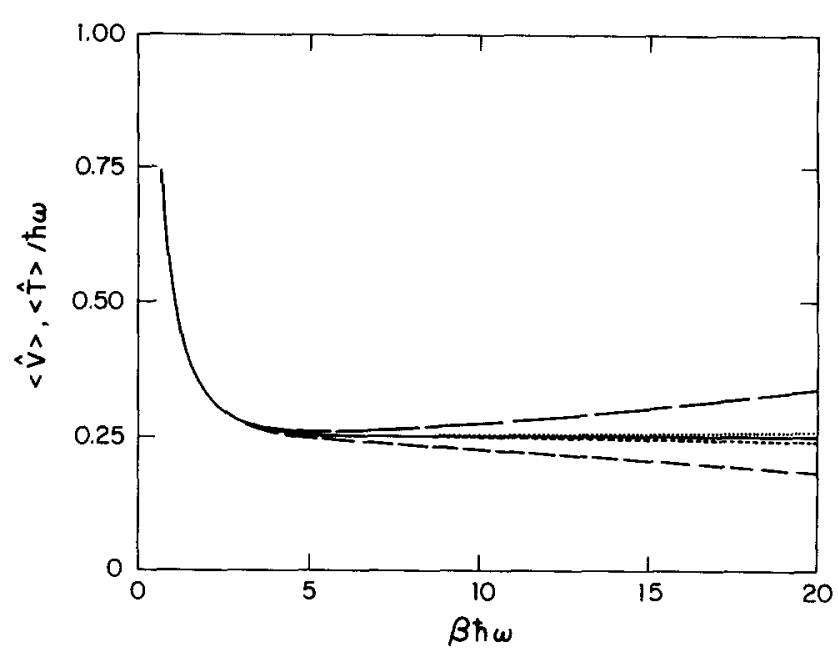

FIG. 4. $\langle V\rangle / \hbar \omega$ and $\langle T\rangle / \hbar \omega$ vs $\beta \hbar \omega$ for the harmonic oscillator system of Sec. VII. Exact result, given by solid line, is compared to $k_{\max }=1=P$ approximations according to various algorithms: $\langle V\rangle / \hbar \omega$ via FPI (shortdash); via trapezoid (medium-dash). $\langle T\rangle / \hbar \omega$ via FPI (dot); via trapezoid (long-dash).

$$
\begin{aligned}
& \frac{1}{\hbar \omega} \cdot \frac{m \omega^{2}}{2}\left\langle\hat{x}^{2}\right\rangle=\frac{\left(f^{P}+1\right) f^{1 / 2}}{2(f+1)\left(f^{P}-1\right)} \\
& \frac{1}{\hbar \omega} \cdot\left\langle\hat{p}^{2}\right\rangle / 2 m \\
& =\beta \hbar \omega / 8 P+\frac{P}{4 \beta \hbar \omega} \\
& \quad \times\left\{\frac{(f-1)\left(f^{2 P-1}+1\right)}{f^{2 P}-1}+\frac{f^{P-1}\left(f^{2}-1\right)}{f^{2 P}-1}\right\} .
\end{aligned}
$$

In Fig. 4 we plot $\langle P E\rangle / \hbar \omega,\langle K E\rangle / \hbar \omega$ for the choice $k_{\max }=10$. Fourier and discretized approximations are compared to the exact result. As can be seen, the FPI results are uniformly superior to their discretized counterparts. This is in contrast to the partition function case where the converse was true. Apparently, the canonical FPI prescription converges quickly to the correct shape, but rather slowly to the correct scale. When the first cumulant partial averaging correction is applied, the (already good) shape of $\rho_{\beta}\left(x_{f}, x_{0}\right)$ is not altered but the erroneous absolute scaling of $\rho_{\beta}$ is renormalized. Of course, if the second cumulant correction to the PA prescription is included in the calculation the shape of $\rho_{\beta}$ (as well as the scale) will be modified. As an illustrative application of the cumulant formalism we will briefly examine the second cumulant correction to $\rho_{\beta}(x, x)$ for the harmonic oscillator.

For the harmonic oscillator it is simple to compute $\int_{0}^{1} d u V(q(u))$, particularly for paths constrained to begin and end at the same point $x=x_{0}=x_{f}$ :

$$
\begin{aligned}
& \frac{2}{m \omega^{2}} \int_{0}^{1} d u V(q(u)) \\
& \quad=x^{2}+2 x \sum_{k=1}^{\infty} a_{k} \frac{\left[1-(-1)^{k}\right]}{\pi k}+\frac{1}{2} \sum_{k=1}^{\infty} a_{k}^{2} .
\end{aligned}
$$

It is then straightforward to perform the Gaussian averages over coefficients $a_{k_{\max }+1}, \ldots, a_{\infty}$ according to Eq. (3.3a). One finds that the right-hand side of Eq. (3.3a) reduces to 
$\rho_{\beta}(x, x)=\rho_{\beta}^{\mathrm{FPI}}(x, x)$

$$
\times \exp \left\{\frac{-(\beta \hbar \omega)^{2}}{12}\left[1-\frac{6}{\pi^{2}} \sum_{k=1}^{k_{\max }} \frac{1}{k^{2}}\right]\right\} e^{\alpha x^{2}+\lambda}
$$

with

$$
\alpha=m \omega^{2} \beta\left(\frac{\beta \hbar \omega}{\pi^{2}}\right)^{2}\left[\frac{\pi^{4}}{24}-\sum_{k=1}^{k_{\max }} \frac{\left[1-(-1)^{k}\right]^{2}}{k^{4}}\right]
$$

and

$$
\lambda=\left[\frac{(\beta \hbar \omega)^{2}}{2 \pi^{2}}\right]^{2}\left[\frac{\pi^{4}}{90}-\sum_{k=1}^{k_{\max }} \frac{1}{k^{4}}\right] .
$$

The factor $\exp \left[\alpha x^{2}+\lambda\right]$ is the second cumulant correction. $\exp [\lambda]$ will modify only $Z_{\beta} ; \exp \left[\alpha x^{2}\right]$ will modify both $Z_{\beta}$ and $\left\langle\hat{x}^{2}\right\rangle$.

To see that the modifications are clearly in the right direction we plot $Z_{\beta}$ vs $\beta \hbar \omega$ in Fig. 5 for the case $k_{\max }=5$, and $\langle P E\rangle / \hbar \omega$ vs $\beta \hbar \omega$ in Fig. 6 for the same value of $k_{\max }$. A lower value of $k_{\max }$ is employed here than was used in the previous figures in order to make the convergence order of the various approximations under study readily apparent. It is easy to see that the second cumulant corrected PA results are uniformly superior to the other approximations discussed in this section.

Partial averaging methods have potential application to the problem of computing finite temperature time correlation functions. As a prototype example of this type of application we consider the problem of computing a thermally averaged rate constant for a simple $1 \mathrm{~d}$ barrier crossing problem. As shown by Miller et al., ${ }^{8}$ the rate constant can be given as

$$
k Q=\int_{0}^{\infty} C_{f}(t) d t,
$$

where $Q$ denotes the partition function for reactants, $k$ is the Boltzmann rate constant, and $C_{f}(t)$ is the flux-flux autocorrelation function. This autocorrelation function can be written (for symmetric barriers) as

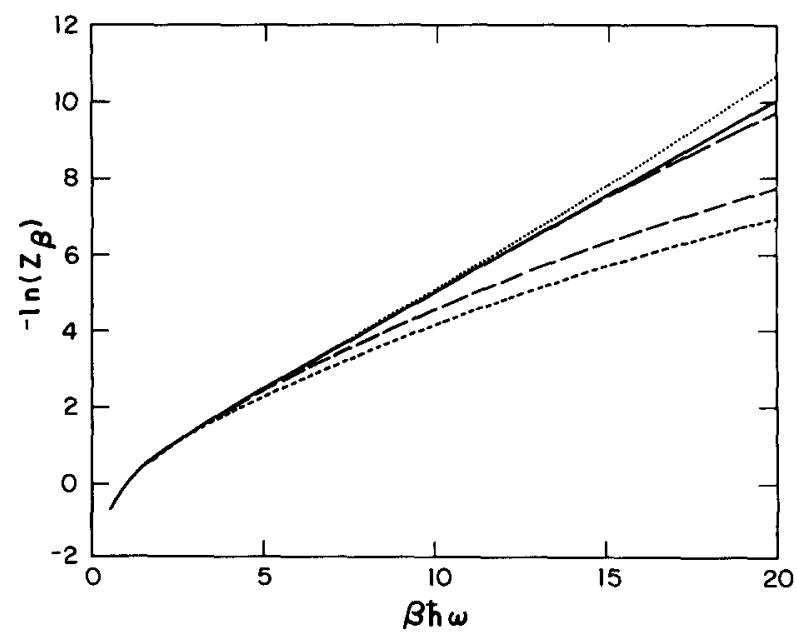

FIG. 5. $-\ln Z_{\beta}$ vs $\beta \hbar \omega$ for the harmonic oscillator system described in Sec. VII. Exact result (solid line) is compared to $k_{\max }=5=P$ canonical FPI (short-dash), PA-FPI (dot), trapezoid (medium-dash), and second cumulant PA-FPI (long-dash) approximations.

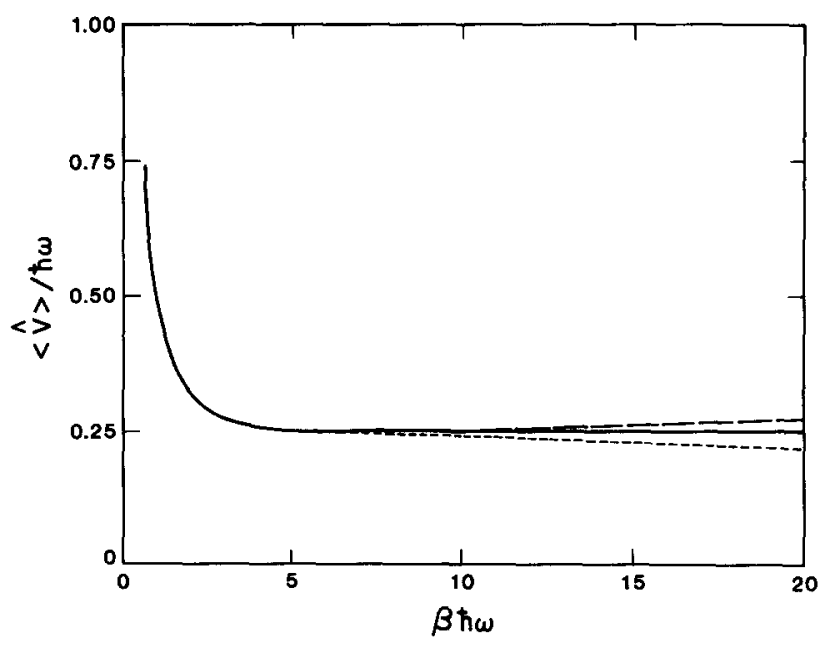

FIG. 6. $\langle V\rangle / \hbar \omega$ vs $\beta \hbar \omega$ for the harmonic oscillator system of Sec. VII. Exact result, given by solid line, is compared to FPI (short-dash), and second cumulant PA-FPI (long-dash) algorithms with $k_{\max }=5$.

$$
C_{f}(t)=\left.\left(\frac{\hbar}{2 m}\right)^{2} \frac{\partial^{2}}{\partial x \partial x^{\prime}}\left|\left\langle x^{\prime}\left|e^{-\beta_{c} \hat{H}}\right| x\right\rangle\right|^{2}\right|_{x^{\prime}=x=0},
$$

where $x=0$ denotes the location of the flux counting surface, and $\beta_{c}$ is given by $\beta_{c}=(\beta / 2+i t / \hbar)$ with $\beta=1 / k_{B} T$.

For purposes of numerical example we consider the calculation of the flux-flux autocorrelation function $C_{f}(t)$ for the model problem of an inverted parabolic barrier, $V(x)=-m \omega^{2} x^{2} / 2$. For this problem $C_{f}(t)$ is available exactly. As shown by Miller et al. ${ }^{8}$

$$
\begin{aligned}
C_{f}(t) & /\left[\frac{k T}{h} \omega\right] \\
= & \frac{\beta \hbar \omega / 2}{\sin (\beta \hbar \omega / 2)} \cdot \frac{\sin ^{2}(\beta \hbar \omega / 2) \cosh \omega t}{\left[\sinh ^{2}(\omega t)+\sin ^{2}(\beta \hbar \omega / 2)\right]^{3 / 2}} .
\end{aligned}
$$

Figure 7 compares this exact result with approximate results in which the Fourier method (with and without partial averaging) was used to compute the relevant density matrix elements [cf. Eqs. (7.1) and (7.2)]. The particular results shown are for $\beta \hbar \omega=5$. The improvement due to partial averaging for a fixed number $\left(k_{\max }\right)$ of Fourier coefficients is impressive. In fact the $k_{\max }=1$ partial averaging results are as good as those produced by $k_{\max }=100$ without partial averaging. Similar improvements were found at other values of $\beta \hbar \omega$. We note in passing that for this problem partial averaging introduces a decaying Gaussian envelope on the flux autocorrelation function.

As a second illustration of the effect of partial averaging on complex time harmonic oscillator motion, we consider the phenomenon of electronic absorption. Suppose that the nuclei of a molecule are initially prepared in a vibrational eigenstate $i$ of a potential surface $V_{l}$. Call this state $|i, l\rangle$. Upon absorption of a photon, the electron cloud of the molecule rearranges so that the nuclei find themselves moving on a different potential surface, $V_{u}$. It is possible to obtain the Franck-Condon absorption spectrum for this process from the time $\operatorname{kernel}^{9 \text { (a) }}$

$$
C(t)=\left\langle i, l\left|e^{-\left(\beta_{a}+i t / \hbar\right) \hat{H}_{u}}\right| i, l\right\rangle,
$$




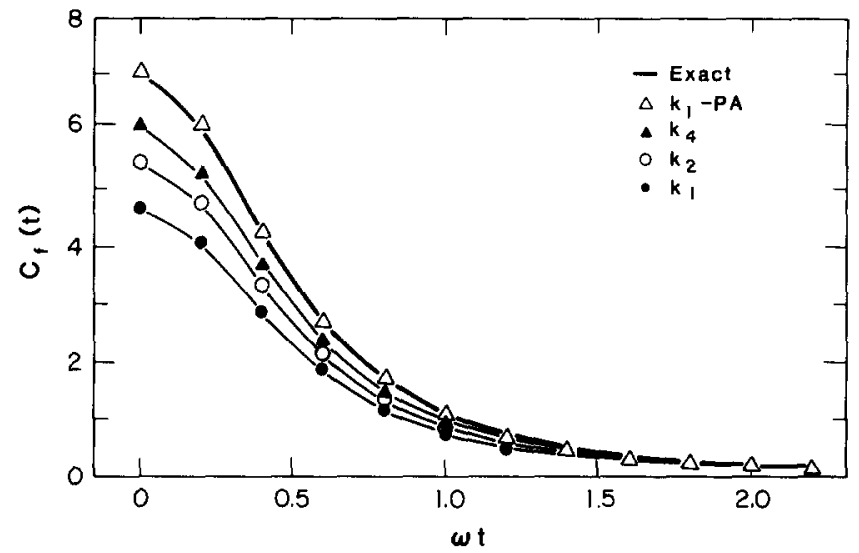

FIG. 7. Plots of flux autocorrelation function $C_{f}(t)$ [Eq. (7.7)] vs $\omega t$ for inverted parabolic barrier described in text at $\beta \hbar \omega=5$. Thick solid line is exact [Eq. (7.8)]. Also shown are canonical FPI approximants for $k_{\max }$ $=1,2,4$, and the $k_{\max }=1 \mathrm{PA}-\mathrm{FPI}$ approximant.

where $H_{u}$ is the Hamiltonian associated with $V_{u}$. In Eq. (7.9), $\beta_{a}$ is an "artificial temperature" parameter. When it is set to 0 , the desired absorption spectrum follows from simple Fourier transformation of $C(t)$. When $\beta_{a}$ is a positive number the Fourier transform of $C(t)$ yields an artificial spectrum, which nevertheless can be easily converted into the correct $\left(\beta_{a} \rightarrow 0\right)$ result. The reason for inserting a nonzero $\beta_{a}$ into the computation when only the $\beta_{a} \rightarrow 0$ limit is physically meaningful stems from the difficulty of evaluating purely real time propagators via MCPI methods. Fortunately, when the potentials involved are harmonic, the integrals entailed by Eq. (7.9) can be performed analytically. Thus, results for any value of $\beta_{a}$ (including $\beta_{a}=0$ ) can be obtained without resorting to Monte Carlo integration techniques. It is then possible to study the effect of PA on the convergence rate of the FPI prescription. In other words, we ask the question: Presuming all integrals involved can be accurately evaluated, how does PA affect the value of $k_{\max }$ needed to pinpoint the function $C(t)$ defined in $\mathrm{Eq}$. (7.9)?

For the case of linearly displaced harmonic surfaces, i.e.,

$$
\begin{aligned}
& V_{l}(x)=\frac{1}{2} m \omega^{2} x^{2}, \\
& V_{u}(x)=\frac{1}{2} m \omega^{2}(x-a)^{2}+V_{0}
\end{aligned}
$$

the previous question can be answered with little effort. Let us concentrate on the simplest case, $i=0$. The essential conclusions we will reach are equally valid for $i>0$, as well as for the finite temperature case, and the case where the electronic transition moment operator (which has been assumed constant here) varies with $x$.

When $i=0$, and for the choice of potentials indicated in Eq. (7.10), we find

$$
\begin{aligned}
C(t)= & e^{-\beta_{c} V_{0}}\left[\frac{m \omega}{\pi \hbar}\right]^{1 / 2} \int d x_{1} d x_{2} e^{-m \omega\left(x_{1}+a\right)^{2} / 2 \hbar} \\
& \times \rho_{\beta_{c}}\left(x_{1}, x_{2}\right) e^{-m \omega\left(x_{2}+a\right)^{2} / 2 \hbar}
\end{aligned}
$$

with $\rho_{\beta_{c}}\left(x_{1}, x_{2}\right)$ the standard propagator for the simple harmonic oscillator potential Eq. (7.10a) and $\beta_{c} \equiv \beta_{a}+i t / \hbar$. The $k_{\max }$ FPI approximation to this propagator function was given in Eq. (7.2), and the PA corrected version in Eq. (7.1). Thus both FPI and PA-FPI approximations to Eq. (7.11) amount to the same $2 \mathrm{~d}$ Gaussian integral, with the PA version further corrected by the familiar scale factor in Eq. (7.1).

We have compared FPI and PA-FPI results for various values of $k_{\max }$ and $\beta_{c}$. [ Exact results may easily be obtained from Eq. (3.3) of Ref. 9(a).] We have found that the rate of convergence with $k_{\max }$ of the two approximations under study is quite insensitive to the value of $\beta_{a}$ used in the calculation. Therefore, we will restrict the following discussion to a single value of $\beta_{a}$. Specifically, we will choose $\beta_{a}=0$, since the exact solution is then particularly simple:

$$
e^{i \omega t / 2} C(t)=e^{-i V_{0} t / \hbar} e^{-b^{2}\left(1-e^{-i \omega t}\right)}
$$

with $b^{2} \equiv m \omega a^{2} / 2 \hbar$. Upon further selecting $V_{0}=-m \omega^{2} a^{2} /$ 2, $C(t)$ becomes extremely easy to describe. For the parameter choices which we will adopt, namely, $\hbar=\omega=m=1$ and $a=3, \operatorname{Re}[C(t)]$ is essentially the Gaussian $\exp \left(-(b \omega t)^{2} / 2\right)$ periodically resurrected at integer multiples of $2 \pi / \omega$. This is shown via the solid line in Fig. 8. Also shown are FPI and PA-FPI approximations for $k_{\max }=5$. Focus first on the FPI (long-dashed line) result. Although this approximation accounts correctly for the initial decay of $C(t)$, successive recurrences damp out rapidly (and incorrectly, of course). On the other hand, the PA-FPI analog (short-dashed line) recurs quite properly at $t=2 \pi$. The second recurrence is less successful, although certainly it is more accurately reproduced than in the simple FPI calculation.

Beyond the second recurrence, the PA result displays pronounced artifactual oscillations. The long time breakdown of PA in this example is easily understood: The magnitude of the PA scale factor blows up at long times as $\exp \left[\omega^{2} t^{2}\left\{1-6 / \pi^{2} \Sigma_{k=1}^{k_{\max }} 1 / k^{2}\right\} / 12\right]$. One therefore expects instability of the overall PA result in this limit. (This is in contrast to the reaction rate example considered above, where the oscillator well was upside down, so that $\omega \rightarrow i \omega$. Or equivalently, the PA scale factor decayed in a Gaussian fashion for large $t$, thus stabilizing the appropriate correlation function.) Equally as important, one is not alarmed by the

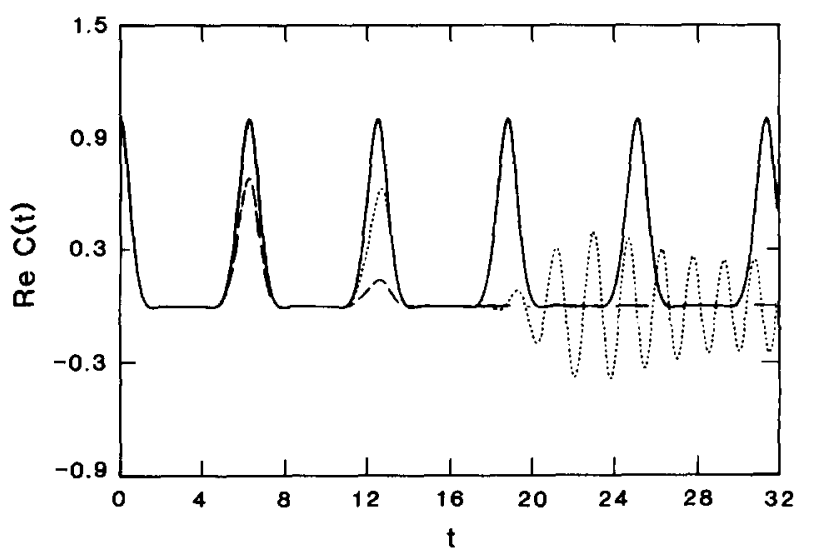

FIG. 8. Real part of time kernel, Eq. (7.50), for linearly displaced oscillator system of Sec. VII and $\beta_{a}=0$. Exact (solid line) result is compared with $k_{\max }=5$ canonical FPI (long-dash) and PA-FPI (short-dash) results. 
instability exhibited in Fig. 8. For one reason, it is easily removed on any time scale of interest. This is illustrated in Fig. 9, where $k_{\max }=25$ results are plotted on the same time interval as before. The PA result is now accurate on nearly the entire interval. (Slight underestimation of the fifth recurrence is discernable, but all spurious oscillations are gone.) The FPI results have also improved, but lag far behind their PA counterparts in accuracy.

From the point of view of presaging MCPI computations of two surface spectra, there is another reason to be pleased with the PA performance: Current technology is confined to relatively short time dynamics. (This restriction stems from the difficulty of computing oscillatory complex time propagator integrals via importance sampling. ${ }^{32}$ ) If we focus on the initial decay and first recurrence of $C(t)$, we find in Fig. 10 that PA dynamics is quite accurate when $k_{\max }$ $=6$. Curiously, the FPI approximation without the PA correction factor stubbornly undershoots the peak at $t=2 \pi$. More than 150 Fourier coefficients are required to obtain the same accuracy as the $k_{\max }=6 \mathrm{PA}$ results.

\section{MCPI DENSITY MATRIX CALCULATIONS FOR ANHARMONIC SYSTEMS}

In this section exploratory MCPI simulations on two anharmonic 1d systems are presented. Specifically, we wish to report on calculations of $\rho_{\beta}(x, x)$ for the Morse well

$$
V(x)=D\left(1-e^{-a x}\right)^{2}
$$

with $D=100.25, a=0.071$, and the double well

$$
V(x)=A \exp \left(-\alpha x^{2}\right)+v_{h 0}
$$

with

$$
v_{h 0}(x)=m \omega^{2} x^{2} / 2,
$$

and $\omega=1, \alpha=4, A=2$. In both systems, $m=\hbar=1$ and $\beta=2$. We have discussed both systems before, ${ }^{7,9(a), 21}$ so the preliminary discussion here will be kept to a minimum. To be compared below are the performances of the canonical FPI and PA-FPI algorithms, as well as analogous results obtained from vaious discretized Cartesian PI algorithms. Specifically, trapezoid rule [based on Eq. (5.1)], midpoint rule [using $\left[V\left(x_{0}\right)+V\left(x_{f}\right)\right] / 2 \rightarrow V\left(\left[x_{f}+x_{0}\right] / 2\right)$ in Eq. (5.1)] and second order effective potential [SOEP, cf. Eq.

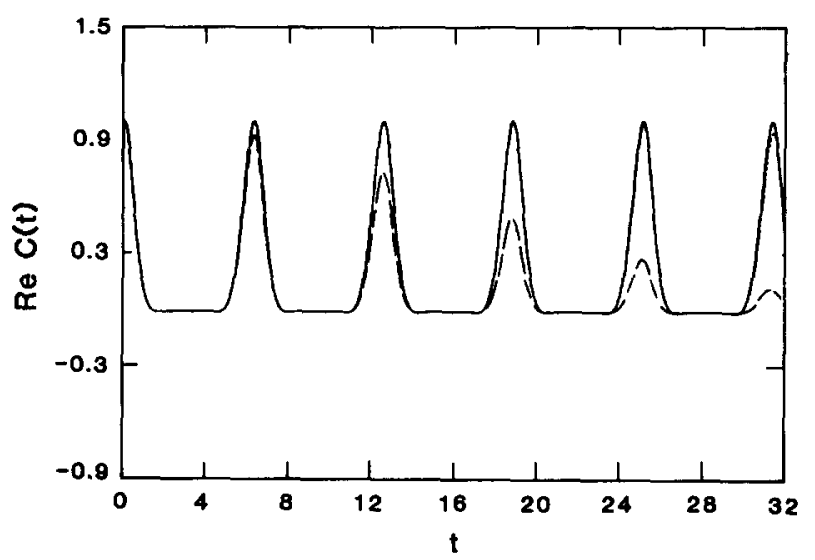

FIG. 9. Same as Fig. 8, except $k_{\max }=25$.

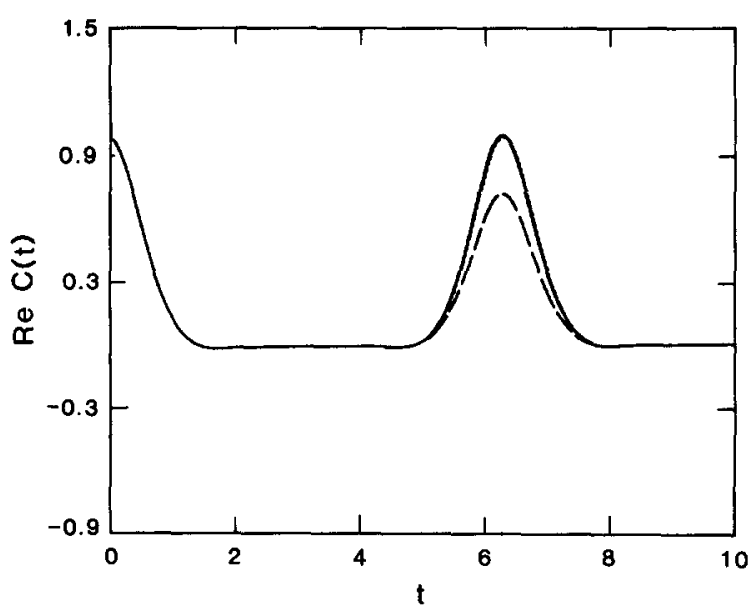

FIG. 10. Same as Fig. 8, except $k_{\max }=6$. Also, note the dilation of time scale relative to Figs. 8 and 9.

(6.5)] variants will be considered. The trapezoid, midpoint and canonical FPI results have been presented before. ${ }^{21}$ They are indicated in the present discussion for convenience in comparing various extant algorithms relevant to the problems under scrutiny. Of the two new methods developed in this paper, the PA-FPI results were obtained in complete analogy to the canonical FPI results obtained in Ref. 21, and the SOEP results in complete analogy to the Trapezoid and Midpoint rule calculations discussed there. We mention here only a few essential details: Four repetitions of each simulation were performed, with 1800 passes per repetition, and the requisite importance sampling was based on the uncoupled Gaussian distributions associated with the kinetic energy part of the Hamiltonian functional. The reader is referred to Ref. 21 for further elaboration upon these remarks.

In Fig. 11 we show results for the Morse system introduced above. The solid line depicts the exact results for

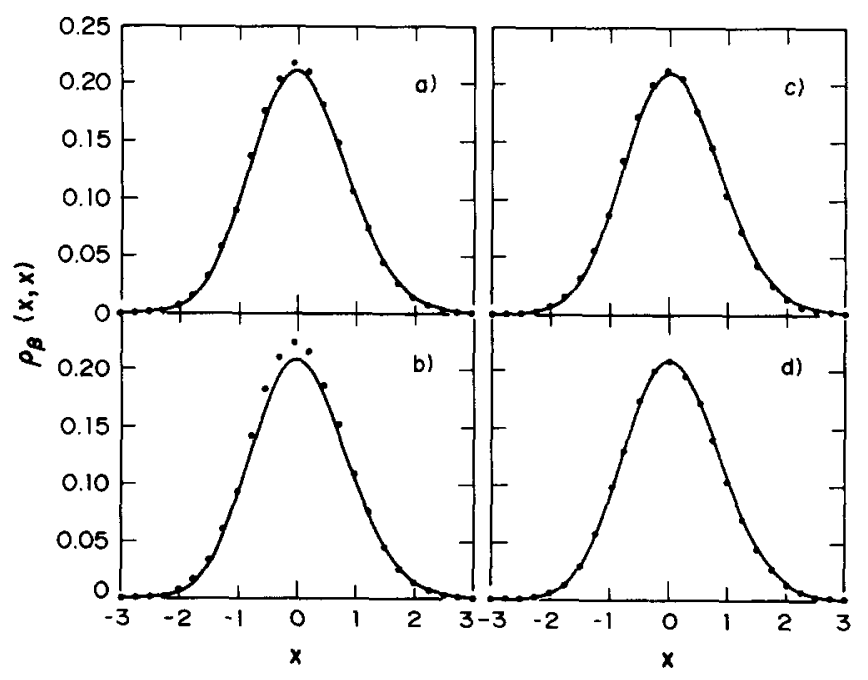

FIG. 11. $\rho_{\beta}(x, x)$ vs $x$ for Morse oscillator system discussed in Sec. VIII. In each of the four panels the solid line depicts exact results. The filled circles are MCPI results, based on (a) $k_{\max }=6$ canonical FPI, (b) $P=6 \mathrm{mid}$ point, (c) $P=6$ trapezoid and (d) $k_{\max }=1$ PA-FPI algorithms. Simulation details are discussed in text. Error bars in general did not exceed the data point circle radius, and therefore have been suppressed. 
$\rho_{\beta}(x, x)$, as obtained by a straightforward eigenvalue/eigenfunction computation..$^{9(a), 21}$ In panels $11(\mathrm{a}), 11(\mathrm{~b})$, and 11 (c) are the canonical FPI, midpoint, and trapezoid results, respectively, for the choice of $k_{\max }=P=6$. [ $P$ here is the Trotter index, or equivalently, the value of the small argument used in all discretized algorithms is given by $\epsilon=\beta /(P+1)$.] Panel $d$ shows the analogous PA-FPI result for $k_{\max }=1$. It is easy to see that in this case one explicit Fourier coefficient in the PA procedure produces results which are considerably more accurate than those achieved with six coefficients in the canonical FPI algorithm. As has been discussed before, ${ }^{21}$ the Trapezoid rule algorithm tends to outperform the canonical FPI algorithm in unnormalized density matrix calculations. Nevertheless, it takes a six coefficient trapezoid rule calculation to (almost) equal the accuracy of a one coefficient PA-FPI analog in the present example.

Figure 12 shows the analogous results for the Gaussian mound potential introduced above. Here, the one coefficient PA-FPI results are clearly superior to any of the simple alternatives implemented at the $k_{\max }=6=P$ level. Finally, we were curious to see how the SOEP algorithm fit into the convergence hierarchy being investigated. We found that about $P=9$ was required to obtain the convergence level observed at the 1 PA-FPI coefficient level. These results are presented in Fig. 13(a). In Fig. 13(b) the analogous $P=9$ trapezoid rule results are shown in order to demonstrate that the improved small argument propagator derived in Sec. VI does indeed produce improved results relative to its predecessor (upon which the trapezoid rule algorithm is based).

\section{PRACTICAL CONSIDERATIONS AND THE ROLE OF FRACTALS IN PATH INTEGRATION}

It has been stressed since the introduction of path integration $^{22}$ that the vast majority of paths which arise in the Trotter product expression for the Cartesian path integral are of the type encountered in Brownian motion. Namely, they are extraordinarily "jagged": If one magnifies a small section which appears smooth, this magnification will reveal
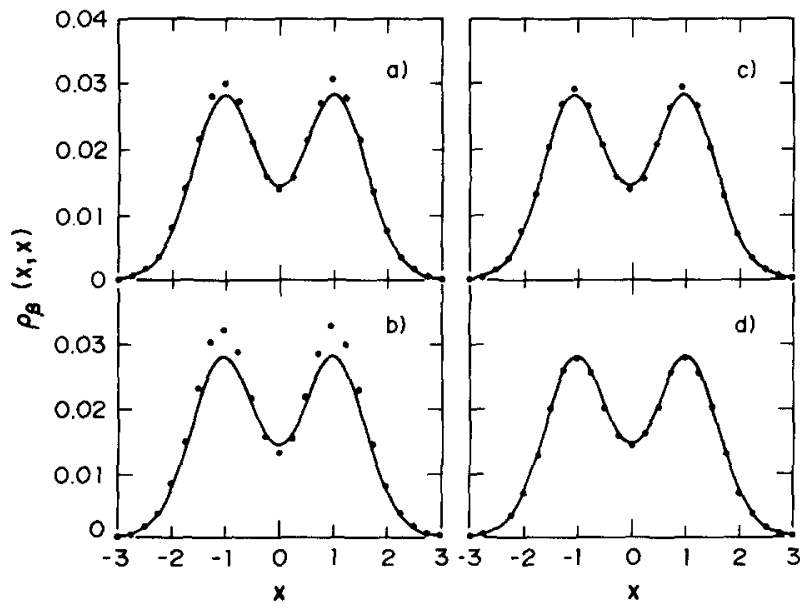

FIG. 12. Same as Fig. 11, except for the Gaussian mound system discussed in Sec. VIII.

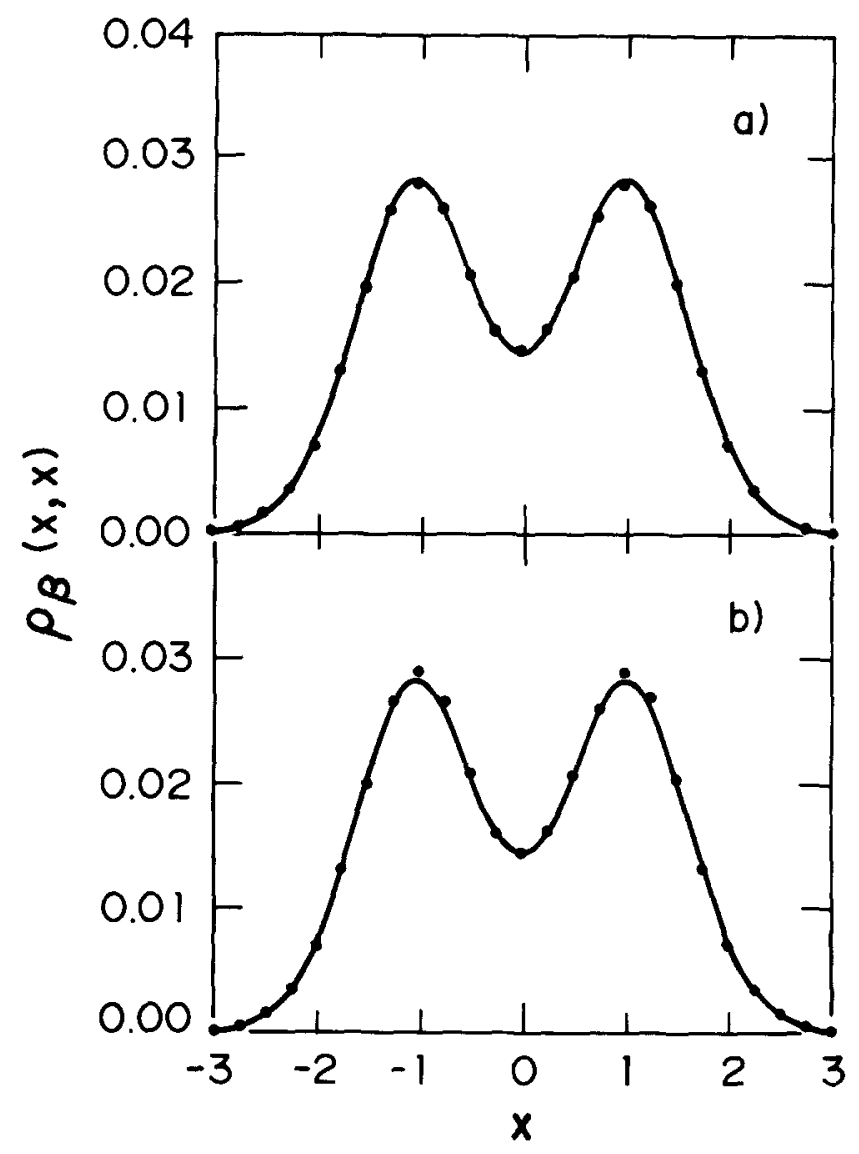

FIG. 13. Effectively, additional panels for the previous figure. In (a) $P=9$ SOEP MCPI results are depicted. In (b) the corresponding $P=9$ trapezoid results are shown.

jagged structure in the small section similar to what had been observed in the complete unmagnified path. Because of the self-similar structure of these objects, they may be characterized as "fractal." ${ }^{23}$ One of their properties is that they are as a rule nowhere differentiable.

In light of these remarks, it would appear foolhardy to attempt to compute quantum mechanical propagators utilizing paths parametrized via low order Fourier series. Such paths are quite smooth (differentiable to all orders) and thus span a phenomenally small subspace of the jagged Brownian paths which in principle contribute to the propagator evaluation. The first indication that such a convergence scheme is not foolhardy, or, in other words, that contributions from smooth paths strongly dominate the PI in spite of their overwhelming paucity, was provided when the FPI representation of the harmonic oscillator problem was formulated. 'As has been seen in Sec. VII, both the statistical density matrix per se, the average static properties dependent on it, and the dynamical correlation functions for harmonic systems can be obtained to high accuracy via finite order Fourier series parametrization of the contributing paths. Precisely how low a value of $k_{\max }$ will give sufficient accuracy depends on the temperature and/or time, the oscillator potential, and the property of interest. Nevertheless, for any specific property, system and conditions, the convergence of the FPI prescription with $k_{\max }$ is found to be monotonic and smooth, ${ }^{24}$ so that some finite value of $k_{\max }$ is guaranteed to suffice. ${ }^{25}$ 
Historically, a second indication that smooth paths dominate simple Cartesian PI's was provided by the success of semiclassical propagator theories of scattering, ${ }^{26}$ tunneling, ${ }^{27,28}$ etc. These analyses hinge upon a steepest descent/ stationary phase evaluation of the propagator, which invariably isolates one or at most a discrete collection of smooth paths, namely, classical mechanical trajectories dictated by the appropriate potential and boundary conditions. The value obtained for the propagator function then follows by appropriately processing the classical trajectory data.

The method of partial averaging provides a third indication of smooth path domination in simple Cartesian PI's. In a sense the lesson here is precisely the same as the one learned in semiclassical propagator techniques: Properly processed smooth trajectory data is all that is needed to obtain accurate values of the propagator function. In partial averaging we select a collection of smooth paths, namely, all paths having the desired end points which can be obtained from a Fourier series of (modest) order $k_{\max }$. Having selected a particular smooth path, we are then faced with the task of summing over higher order fluctuations (Fourier modes with $k>k_{\max }$ ). Just as in semiclassical theories, these higher order fluctuations are not completely neglected, but rather summed over in an approximate way. Indeed, the same assumption motivates the approximations employed in the two procedures (i.e., the fluctuations are presumed small). One finally arrives in PA theory at a simple prescription for processing the smooth path data in an optimal way $\left(\int_{0}^{1} d u V_{\text {eff }}\left(q_{k_{\max }}(u), u\right)\right)$. As we have seen in the previous sections, with this processing procedure very smooth paths are sufficient to obtain converged quantum mechanical information about a variety of systems under a variety of conditions. To make this notion clearer, we depict in Fig. 14(a) a typical $k_{\max }=1$ Fourier path for the inverse temperature $\beta=2\left(x_{0}=x_{f}=m=\hbar=1\right)$. Also, depicted in Fig. 14(a) are $V(q(u))$ and $V_{\text {eff }}(q(u), u)$ for the Gaussian mound potential discussed in Sec. VIII. Recall that a $k_{\max }=1$ description of paths proved sufficient for quantitative evaluation of $\rho_{\beta}(x, x)$ at $\beta=2$, provided $V_{\text {eff }}(q(u), u)$ was employed in the necessary "action integral." This amounted to a particular and apparently optimal processing of the smooth trajectory data contained in $k_{\max }=1$ paths [i.e., the solid line in Fig. 14(a) is transformed into the short-dashed line in that figure]. An example of improper or nonoptimal path processing is provided by the canonical FPI procedure (the solid line in Fig. 14(a) is transformed into the long-dashed line there). If this processing procedure is employed the results are dramatically worse than those shown in Fig. 12(d). [Note: $k_{\max }=1$ results for the canonical FPI algorithm have not been included in the figures. However, from the $k_{\max }=6$ results shown in Fig. 12(a), the veracity of the present claim can be inferred.]

To address the "practical considerations" alluded to in the current section heading, we have plotted in Fig. 14(b) analogous results for a typical $k_{\max }=10$ path (again, the solid line). At the level of $k_{\max }=10$ the canonical FPI algorithm results are approaching the desired limit (note that the proper vs improper processing procedures, i.e., short-dashed vs long-dashed lines, are nearly coincident). We wish to stress here that substantial savings in computational effort results from the order of magnitude reduction in the number of Fourier coefficients enabled by PA. First, fewer integration variables need to be sampled (in the present case, ten times fewer). Moreover, the number of quadrature points needed for the action integral computation, and hence the number of (expensive) calls to the system potential, are significantly reduced when they are based on a low order Fourier path [cf. the short-dashed line in Fig. 14(a) vs the longdashed line in Fig. 14(b) ]. Overall, the effort needed to converge a $k_{\max }=1$ calculation in our current example is nearly an order of magnitude smaller than that needed to converge a $k_{\max }=10$ calculation without PA. Although the relative effort needed to converge results with and without PA will clearly be system dependent, it is heartening to see that for the nontrivial system just discussed, PA proves itself to be a considerable asset.
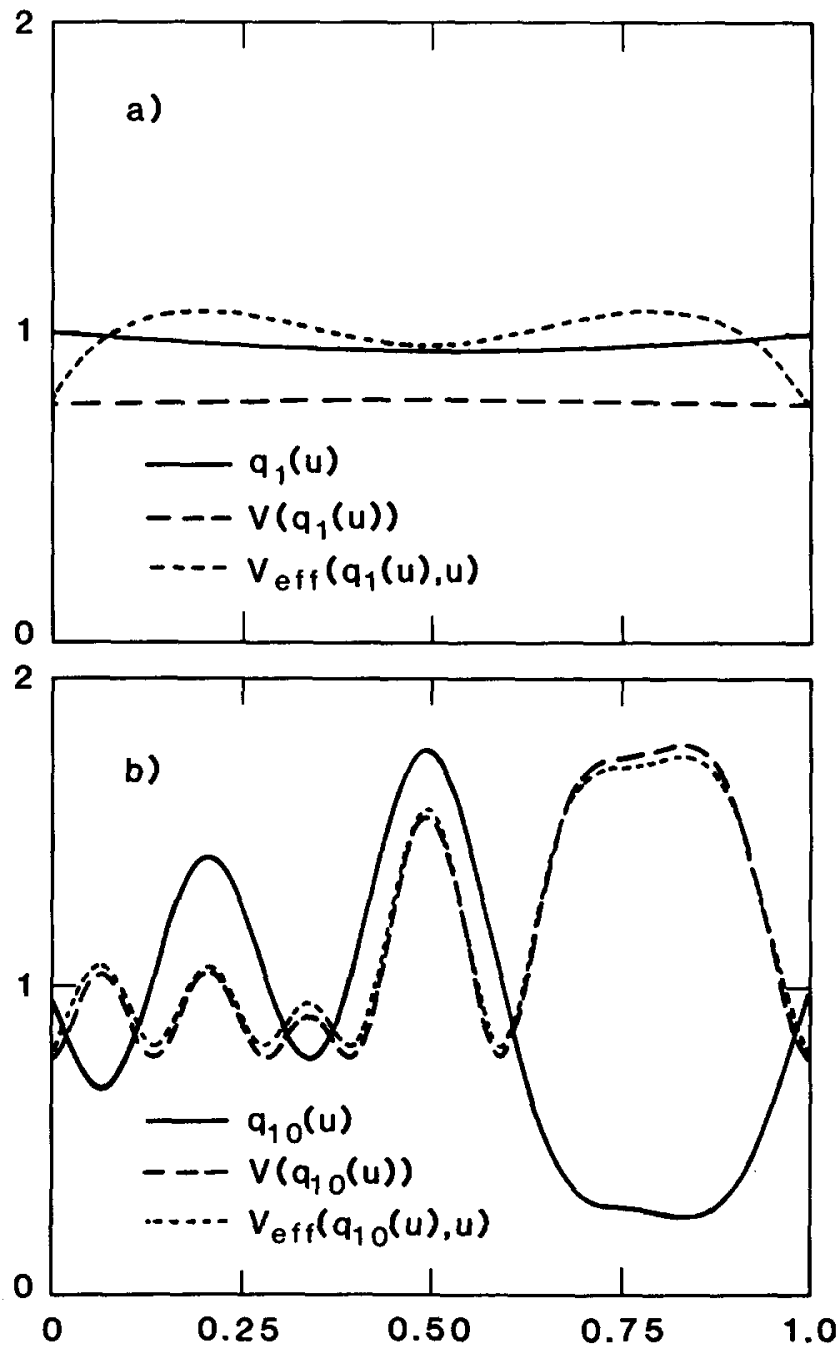

$\mathbf{u}$

FIG. 14. The solid lines in Figs. 14(a) and 14(b) depict typical $k_{\max }=1,10$ paths, respectively, for the choice of parameters $1=x_{0}=x_{f}=\hbar=m=\beta$ । 2. The long-dashed line in each panel indicated $V(q(u))$ for the path $q(u)$ shown in that panel, with $V$ the Gaussian mound potential of Sec. 8. The short-dashed line is a plot of the corresponding PA effective potential $V(q(u), u)$ 


\section{DISCUSSION AND CONCLUSION}

In this paper we have developed a new scheme for computing Cartesian path integrals. This formulation starts with the Fourier coefficient representation of the sum over paths. It then removes the small, rapid fluctuations contributed by high order terms in the Fourier series parametrization of paths in a way which is both physically appealing and mathematically sound. In its simplest manifestation, the procedure leads to a prescription which we have termed partial averaging. The partial averaging prescription is simply a canonical Fourier path integral (FPI) description with the system potential replaced by an effective "time dependent" potential. We have examined convergence rates of the canonical FPI algorithm vs the partial averaging modified FPI algorithm (PA-FPI) for both harmonic and anharmonic systems undergoing both imaginary and complex time evolution. Partial averaging has proven uniformly effective in improving the performance of the canonical FPI algorithm. Thus, as a general rule, PA-FPI would appear to supplant its canonical counterpart as the Fourier algorithm of choice.

Also considered in our convergence investigations were various standard discretized algorithms. It is now reasonably well understood ${ }^{21}$ that these are in many respects variants of the canonical FPI algorithm (or vice versa). The respective positions of canonical FPI and discretized algorithms in convergence hierarchies depends on the property of interest, as was documented in some detail for the case of harmonic systems in Sec. VII. However, the PA-FPI algorithm demonstrated convergence characteristics superior to all discretized algorithms for the rather wide variety of properties considered in Secs. VII and VIII.

For motion which takes place in a single (multidimensional) potential well the FPI-PA method seems ideally suited. The discussion of Sec. VII showed partial averaging to be quite successful in the treatment of harmonic systems. The results of Sec. VIII then confirmed that this success is not threatened when the single well is anharmonic (Morse case) or a modest bump is put in it (Gaussian mound case). In all three cases we suspect that free-particle paths sample the path space rather efficiently, and that the small rapid oscillations associated with high order Fourier coefficients are efficiently averaged out by partial averaging.

In potentials which admit tunneling between two deep wells the situation is less clear. While there is no reason to believe that the PA-FPI prescription would not converge rapidly if we could easily compute the multidimensional integrals which arise at each stage of the convergence procedure, current Fourier methods may be inefficient for importance sampling evaluation of these integrals. Free-particle paths are rather far removed in character from the kink-like paths which are known to give dominant contributions to path integrals for these systems. ${ }^{22(b)}$ Work is currently underway to devise more natural sampling schemes for this important and still controversial ${ }^{29}$ class of processes.

\section{ACKNOWLEDGMENTS}

We wish to thank L. Pratt and K. McDowell for helpful comments concerning this manuscript. Work at the University of Rhode Island was supported in part by grants from the University of Rhode Island Computer Center, Research Corporation and the University of Rhode Island Engineering Computer Laboratory.

\section{APPENDIX}

We wish to derive the complex time Fourier PI here. Our starting point is the small argument propagator for $\epsilon \equiv \beta_{\mathrm{c}} / N$ :

$\rho_{\epsilon}\left(x_{f}, x_{0}\right)=\left[\frac{m}{2 \pi \hbar^{2} \epsilon}\right]^{1 / 2} \exp \left\{\frac{-m}{2 \hbar^{2} \epsilon}\left(x_{f}-x_{0}\right)^{2}-\epsilon V\left(x_{f}\right)\right\}$.

It is then easy to construct a Trotter product approximation to the finite argument case:

$$
\begin{aligned}
\rho_{\kappa}\left(x_{f}, x_{0}\right)= & \left\langle x_{f}\left|\left(e^{-\epsilon \widehat{H}}\right)^{N}\right| x_{0}\right\rangle \\
\cong & A_{N} \int d x_{1} \ldots d x_{N-1} \exp \left\{\frac{-m}{2 \hbar^{2} \epsilon} \sum_{j=1}^{N}\left(x_{j}-x_{j-1}\right)^{2}\right. \\
& \left.-\epsilon \sum_{j=1}^{N} V\left(x_{j}\right)\right\},
\end{aligned}
$$

where $x_{N} \equiv x_{f}$ and $A_{N} \equiv\left[m / 2 \pi \hbar^{2} \epsilon\right]^{N / 2}$. The approximation just recorded tends toward equality as $N \rightarrow \infty$.

Using Feynman's sum over histories interpretation of this Trotter product formula, it is possible to regard in the $N \rightarrow \infty$ limit

$$
\begin{aligned}
& \sum_{j=1}^{N}\left(x_{j}-x_{j-1}\right)^{2}=\delta \int_{0}^{\gamma} d s \dot{q}(s)^{2}, \\
& \sum_{j=1}^{N} V\left(x_{j}\right)=\frac{1}{\delta} \int_{0}^{\gamma} d s V(q(s)),
\end{aligned}
$$

where $\gamma$ is an arbitrary positive number $\delta \equiv \gamma / N$ and $q(s)$ is the "trajectory" limit of the jagged path determined by a particular configuration $\left(x_{0}, \ldots, x_{N}\right)$. Since the value of $\gamma$ is arbitrary it must disappear from the final result. To see how this occurs, we take the ratio of $\rho_{\beta_{\mathrm{c}}} / \rho_{\beta_{c}}^{f_{c}}, \rho_{\beta_{c}}^{f^{p}}\left(x_{f}, x_{0}\right)$ being the free particle propagator for complex $\beta_{c}$. Because $A_{N}$ is potential independent it cancels out of the ratio and we find

$$
\frac{\rho_{\beta_{c}}\left(x_{f}, x_{0}\right)}{\rho_{\beta_{c}}^{f}\left(x_{f}, x_{0}\right)}=\frac{\Sigma_{\text {paths }} \exp \left[\left(-m \gamma / 2 \hbar^{2} \beta_{c}\right) \int_{0}^{\gamma} d s \dot{q}^{2}(s)-\left(\beta_{c} / \gamma\right) \int_{0}^{\gamma} d s V(q(s))\right]}{\Sigma_{\text {paths }}\left[\exp \left(-m \gamma / 2 \hbar^{2} \beta_{c}\right) \int_{0}^{\gamma} d s \dot{q}^{2}(s)\right]},
$$

Having expressed the path integral in a representation independent form, we next seek a particular representation convenient to our present goals. To wit, we use a Fourier representation for the paths

$$
q(s)=x_{0}+\left(x_{f}-x_{0}\right) s / \gamma+\sum_{k=1}^{\infty} a_{k} \sin (k \pi s / \gamma)
$$

and sum over paths by integrating over Fourier coefficients, so that 


$$
\frac{\rho_{\beta_{c}}\left(x_{f}, x_{0}\right)}{\rho_{\beta_{c}}^{f p}\left(x_{f}, x_{0}\right)}=\frac{s d a_{1} \ldots d a_{\infty} \exp \left[\left(-m \gamma / 2 \hbar^{2} \beta_{c}\right) \int_{0}^{\gamma} d s \dot{q}^{2}(s)-\left(\beta_{c} / \gamma\right) \int_{0}^{\gamma} d s V(q(s))\right]}{\int d a_{1} \ldots d a_{\infty} \exp \left[\left(-m \gamma / 2 \hbar^{2} \beta_{c}\right) \int_{0}^{\gamma} d s \dot{q}^{2}(s)\right]} .
$$

It is trivial to integrate $\int_{0}^{\gamma} d s \dot{q}^{2}(s)$, which results in

$$
\frac{\rho_{\beta_{c}}\left(x_{f}, x_{0}\right)}{\rho_{\beta_{c}}^{f p}\left(x_{f}, x_{0}\right)}=\frac{\int d a_{1} \ldots d a_{\infty} \exp \left\{-\Sigma_{k=1}^{\infty}\left[m(\pi k)^{2} / 4 \hbar^{2} \beta_{c}\right] a_{k}^{2}-\left(\beta_{c} / \gamma\right) \int_{0}^{\gamma} d s V(q(s))\right\}}{\int d a_{1} \ldots d a_{\infty} \exp \left\{-\Sigma_{k=1}^{\infty}\left[m(\pi k)^{2} / 4 \hbar^{2} \beta_{c}\right] a_{k}^{2}\right\}},
$$

Finally, changing variables in the potential integral according to $s=\gamma u$ brings us to our final expression, Eq. (4.1) of the text. Note that $\gamma$ has vanished from the final result, as anticipated.

${ }^{\prime}$ R. P. Feynman and A. R. Hibbs, Quantum Mechanics and Path Integrals (McGraw-Hill, New York, 1965).

${ }^{2}$ N. Metropolis, A. W. Rosenbluth, M. N. Rosenbluth, A. H. Teller, and E. Teller, J. Chem. Phys. 21, 1087 (1953).

${ }^{3}$ M. Suzuki, Prog. Theor. Phys. 56, 1454 (1976); Commun. Math. Phys. 51, 183 (1976); 57, 193 (1977).

${ }^{4}$ H. De Raedt and B. De Raedt, Phys. Rev. A 28, 3575 (1983).

${ }_{5}^{5}$ D. Thirumalai and B. Berne, J. Chem. Phys. 79, 5029 (1983).

${ }^{6}$ K. S. Schweizer, R. M. Stratt, D. Chandler, and P. G. Wolynes, J. Chem. Phys. 75, 1347 (1981).

${ }^{7}$ J. D. Doll, R. D. Coalson, and D. L. Freeman, Phys. Rev. Lett. 55, 1 (1985).

${ }^{8}$ W. H. Miller, S. D. Schwartz, and J. W. Tromp, J. Chem. Phys. 79, 4889 (1983).

${ }^{9}$ (a) R. D. Coalson, J. Chem. Phys. 83, 688 (1985); (b) D. Thirumalai and B. Berne, Chem. Phys. Lett. 116, 471 (1985).

${ }^{10}$ (a) J. D. Doll and L. E. Myers, J. Chem. Phys. 71, 2880 (1979); (b) J. D. Doll and D. L. Freeman, ibid. 80, 2239 (1984); (c) D. L. Freeman and J. D. Doll, ibid. 80, 5709 (1984).

"J. D. Doll, J. Chem. Phys. 81, 3536 (1984).

${ }^{12}$ S. O. Rice, in Selected Papers on Noise and Stochastic Processes, edited by N. Wax (Dover, New York, 1954).

${ }^{13}$ S. Y. Lee and E. J. Heller, J. Chem. Phys. 76, 3035 (1982); R. D. Coalson and M. Karplus, Chem. Phys. Lett. 90, 301 (1982).

${ }^{14}$ N. Corbin and K. Singer, Mol. Phys. 46, 671 (1982).

${ }^{15}$ R. P. Feynman, Statistical Mechanics (Benjamin, Reading, Mass., 1972).

${ }^{16}$ R. Kubo, J. Phys. Soc. Jpn. 17, 1100 (1962); N. Van Kampen, Phys. Rep. 24, 171 (1976).

${ }^{17}$ R. D. Coalson (unpublished results).

18J. G. Kirkwood, Phys. Rev. 44, 31 (1933); 45, 116 (1934).

${ }^{19}$ Actually, we have not proved that terms $\beta^{2}$ with $z>0$ but not an integer do not occur. However, an appeal to the Wigner-Kirkwood expansion enables us to leave out such terms with confidence. In any case, the important point for present purposes is simply that $c_{0}=0$ on the right-hand side of Eq. (6.2).

${ }^{20}$ It is somewhat unusual to propagate wave packets under the imaginary time Schrödinger equation. However, it is not unheard of. For example, the calculation of finite temperature correlation functions (infrared and two-surface) can be implemented by propagating localized wave packets alternately in real and imaginary time. [Cf. J. R. Reimers, K. R. Wilson, and E. J. Heller, J. Chem. Phys. 79,4749 (1983).] Also, an initially localized probability distribution can be evolved under the Schmoluchowski equation by propagating a localized wave packet under the appropriate imaginary time Schrödinger equation. [Cf. A. N. Drozdov, V. Zitserman, and V. F. Baibuz, Chem. Phys. 88, 81 (1984).] Finally, the recalcitrant reader can simply replace $\epsilon \rightarrow i \epsilon / \hbar$ everywhere in this section to obtain the analogous real time results. All conclusions concerning the accuracy of the improved real time propagators thus obtained follow via reasoning identical to that being used in our discussion of imaginary time evolution. ${ }^{2}$ 'R. D. Coalson, J. Chem. Phys. 85, 926 (1986).

${ }^{22}$ (a) See, for example, Fig. 7-1 of Ref. 1; (b) M. Creutz and B. Freedman, Ann. Phys. (N.Y.) 132, 427 (1981).

${ }^{23} \mathrm{~B}$. Mandelbrot, The Fractal Geometry of Nature (Freeman, San Francisco, 1982).

${ }^{24}$ In the case of long time evolution, a transient region where convergence is not monotonic may have to be traversed before this desirable behavior sets in.

${ }^{25}$ For example, in the case of thermodynamic properties, the oscillator is forced entirely into its ground state when $\beta \hbar \omega \gtrsim 5$. Thus, no further thermodynamic information is obtained by considering $\beta \hbar \omega$ greater than this value. From Figs. 1 and 2 it is clear that all thermodynamic and static finite temperature properties can be obtained from the canonical FPI prescription with $k_{\max }<10$.

${ }^{26}$ W. H. Miller, Adv. Chem. Phys. 25, 69 (1974).

${ }^{27}$ J. S. Langer, Ann. Phys. (N.Y.) 41, 108 (1967).

${ }^{28}$ S. Coleman, Phys. Rev. D 15, 2929 (1977).

${ }^{29}$ R. M. Stratt, Phys. Rev. Lett. 55, 1443 (1985), and references therein.

${ }^{30}$ W. H. Miller, J. Chem. Phys. 63, 1166 (1975).

${ }^{31}$ By "linear exponentials" we mean functions of the form $\exp \{\boldsymbol{\gamma} \cdot \mathrm{x}\}$, where $\mathbf{x}$ is the $d$ dimensional Cartesian coordinate vector as defined in Sec. II and $\gamma$ is a vector of scale constants. We wish to distinguish such functions from radial exponentials $\exp \{\gamma r\}$, with $r$ the three-dimensional distance between two particles. Radial exponential functions, which arise in the study of quantum fluids, are not strictly Gaussian transformable.

${ }^{32}$ It is important to stress that the partial averaging procedure does not completely eliminate this difficulty. It would, however, appear to lessen the problem since fewer integration variables suffice to obtain converged results. One can then afford to sample these variables more vigorously in order to reduce statistical error bars to a tolerable size. 
The Journal of Chemical Physics is copyrighted by the American Institute of Physics (AIP). Redistribution of journal material is subject to the AIP online journal license and/or AIP copyright. For more information, see http:/ojps.aip.org/jcpo/jcpcr/jsp Copyright of Journal of Chemical Physics is the property of American Institute of Physics and its content may not be copied or emailed to multiple sites or posted to a listserv without the copyright holder's express written permission. However, users may print, download, or email articles for individual use. 
The Journal of Chemical Physics is copyrighted by the American Institute of Physics (AIP). Redistribution of journal material is subject to the AIP online journal license and/or AIP copyright. For more information, see http://ojps.aip.org/jcpo/jcper/jsp 